\title{
NURSE PRACTITIONERS AND EMEDREC: A PHENOMENOLOGICAL EXPLORATION
}

by

\author{
Nadine Medley \\ Bachelor of Science in Nursing, Ryerson University, 2012
}

A thesis presented to Ryerson University

in partial fulfillment of the

requirements for the degree of

Master of Nursing

in the Program of

Nursing

Toronto, Ontario, Canada, 2017

(C) Nadine Medley, 2017 


\section{AUTHOR'S DECLARATION FOR ELECTRONIC SUBMISSION OF A THESIS}

I hereby declare that I am the sole author of this thesis. This is a true copy of the thesis, including any required final revisions, as accepted by my examiners.

I authorize Ryerson University to lend this thesis to other institutions or individuals for the purpose of scholarly research.

I further authorize Ryerson University to reproduce this thesis by photocopying or by other means, in total or in part, at the request of other institutions or individuals for the purpose of scholarly research.

I understand that my thesis may be made electronically available to the public. 


\title{
NURSE PRACTITIONERS AND EMEDREC: A PHENOMENOLOGICAL EXPLORATION
}

Nadine Medley

Master of Nursing, Ryerson University, 2017

\begin{abstract}
The impact of technology on patient safety has been inconclusive. A qualitative approach informed by Van Manen $(1990 ; 2014)$ has the potential to reveal nuances inherent in the process of technology use in healthcare; therefore, this study's purpose was to understand and assign meaning to the lived experience of Nurse Practitioners' (NP) eMedRec use. Data were collected via two interviews per participant with a total sample of six NPs. A layered approach was used for data analysis including epoche-reduction, thematic analysis and cognitive mapping. The major themes identified were: 1) Caring for the patient and family, 2) Enacting patient safety, 3) Practicing within the professional role and scope, 4) Wading through the system and working through the process, and 5) Learning and unlearning overtime. Key recommendations are that eMedRec systems could better prioritize patients and be designed in consultation with the NPs, patients and their families.
\end{abstract}




\section{ACKNOWLEDGEMENTS}

I would like to acknowledge the contributions of my thesis committee: Dr. Nancy Purdy RN, PhD, Dr. Oona St. Amant RN, PhD, Dr. Jennifer Stinson RN, PhD and Dr. Patrick Neumann Eur. Eng. PhD. I sincerely appreciate how you shared your expertise with me to ensure that this research study was scholarly and methodologically sound. Your guidance throughout all phases of the research process has proven invaluable. To my co-supervisors, Nancy and Oona I will be forever grateful for not only your academic expertise and guidance, but your empathy during the challenging moments and inspiration to push me to excel beyond what I thought I was capable of. I would also like to thank Richard Wray, Helen Edwards, Trey Coffey and Mary McAllister for their direction during the planning phases of this study and their on-going support.

I am extremely grateful to Ryerson University for the funding I received in support of my thesis completion and to the Registered Nurses Foundation of Ontario (RNFOO) for awarding me the 2016 ONIG scholarship from the Ontario Nursing Informatics Group. I attribute much of my success in this program to the funding as well as the academic support I received over the course of my program. A sincere thank you to everyone. 


\section{DEDICATION}

This Master's thesis is dedicated to my late grandmother, Daisy Medley, for inspiring me to pursue higher education and for being a quiet, yet powerful source of change in the lives of the women in my family. I am eternally grateful for your love and inspiration. Also to my parents, Pauline and Earl Medley and my brother, Anthony Medley, your love and support sustained me during this challenging process. I could never repay you for all you have done.

Finally, to the expert Nurse Practitioners who contribute greatly to lives of the patients and families they serve. Thank you for sacrificing your time to speak with me. I endeavoured to represent your collective voices and tell your stories to reveal the complexities of your professional experiences with eMedRec. Thank you for your contributions. 


\section{Table of Contents}

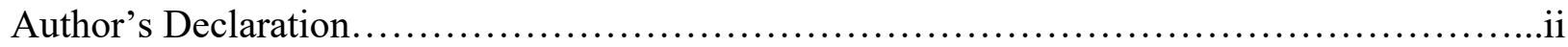

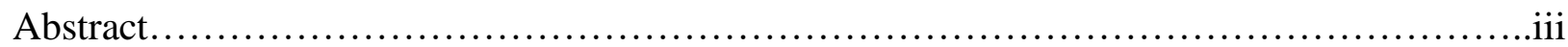

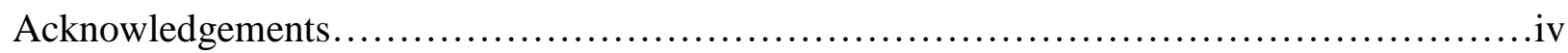

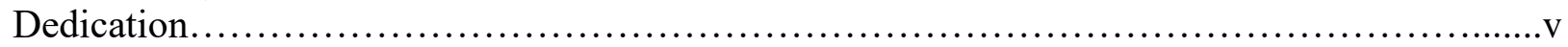

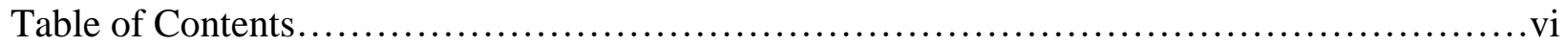

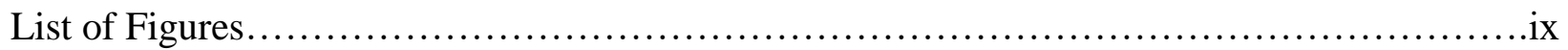

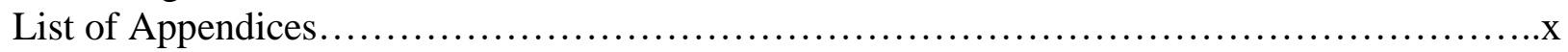

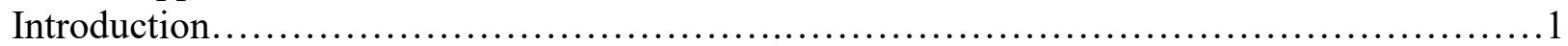

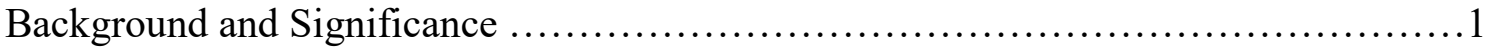

Study Purpose and Question............................................................

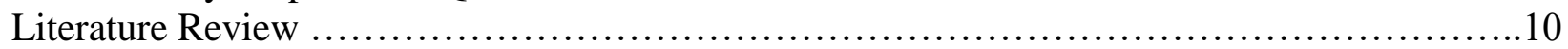

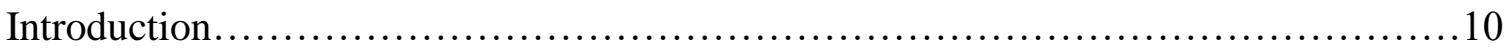

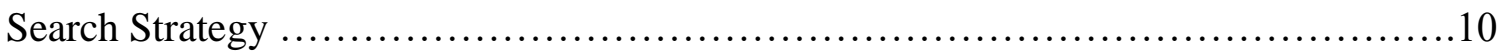

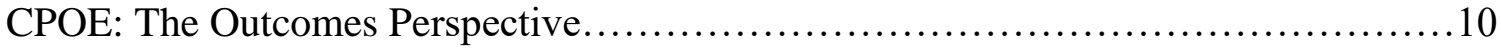

Technology Usability and User Experience in Healthcare ...........................13

Theories of Human-Computer Interaction and the Complex System ....................17

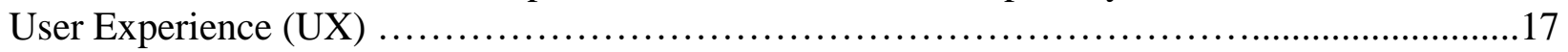

Technology Acceptance ....................................................... 19

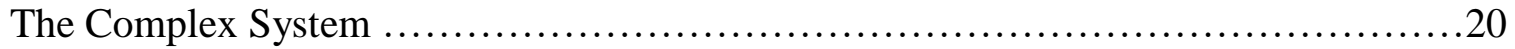

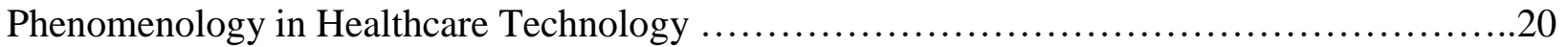

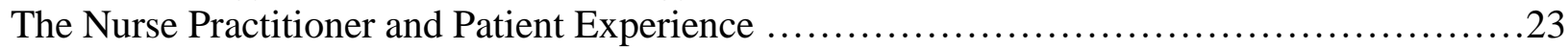

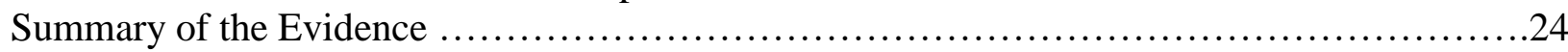

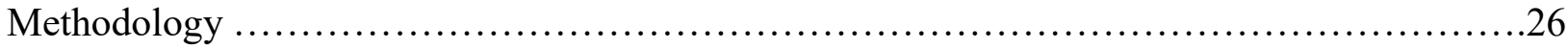

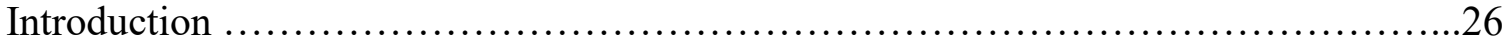

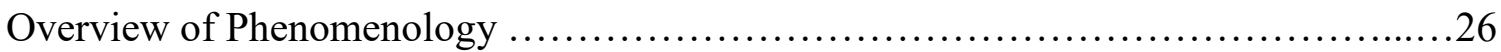

Husserl's Phenomenology ............................................2

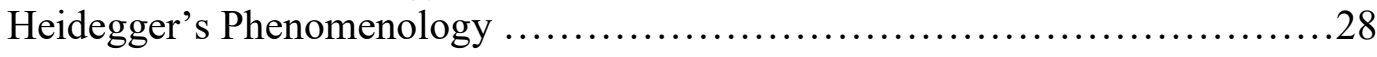

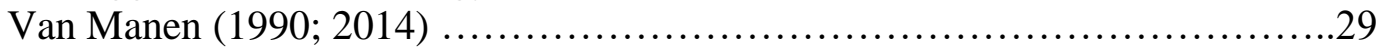

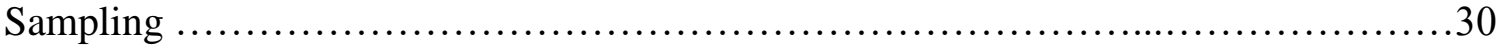

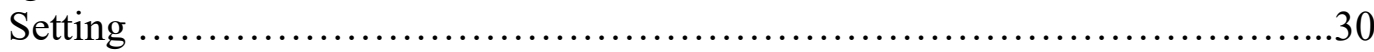

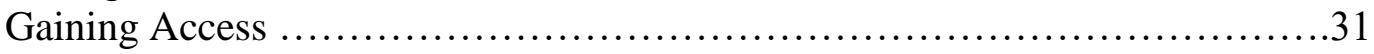

Sample................................................................ 31

Sample Population - NP and MD Role in eMedRec ….....................32

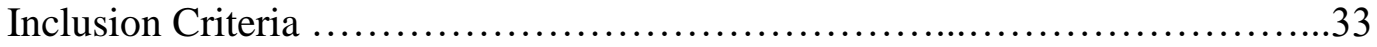

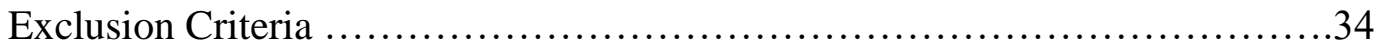

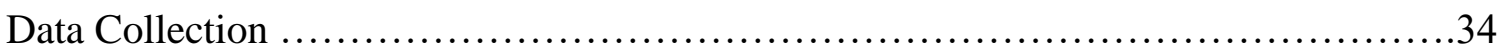

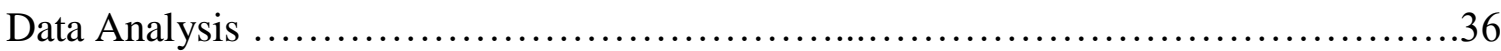

Epoche and Phenomenological Reduction ...................................37

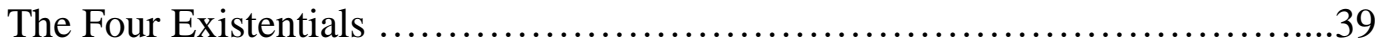

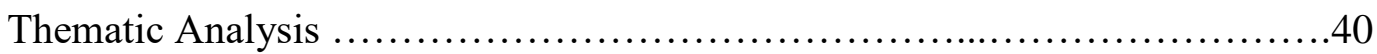

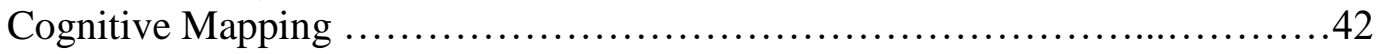

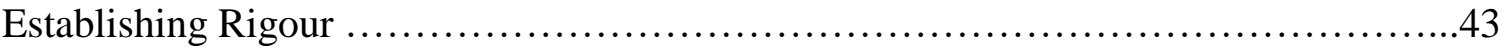

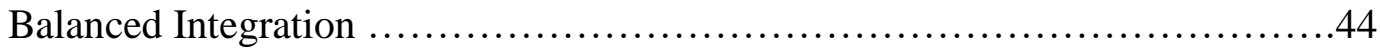

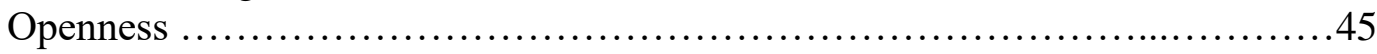


Concreteness .......................................................45

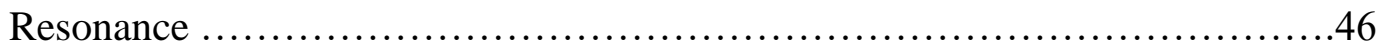

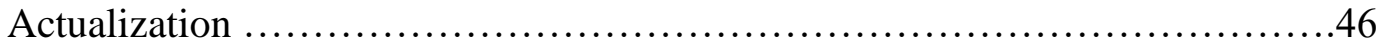

Reflexivity ................................................................ 47

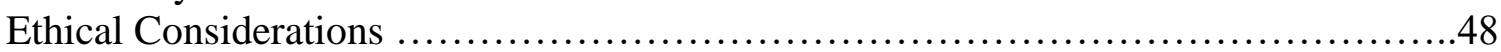

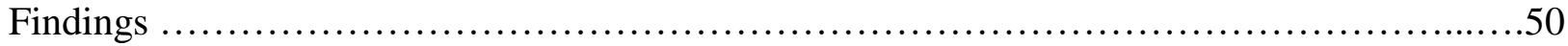

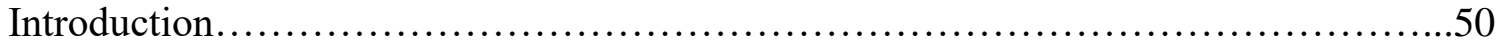

Theme one: Caring for the Patient and Family ....................................51

Sub-theme: Adapting to the Complexity of Patient Care .....................552

Sub-theme: Communicating with the Patient, Family and HCPs ................57

Sub-theme: Being Accountable for Patient Care ...........................55

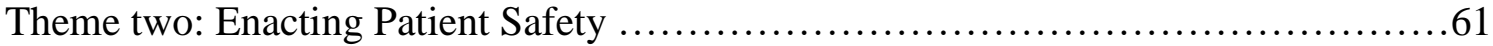

Sub-theme: Double-checking as a Reflection of "Good" Care .................662

Sub-theme: Correcting Errors ............................................63

Sub-theme: Working Around Obstacles ..................................65

Theme three: Practicing within the Professional Scope and Role ....................66

Sub-theme: Coordinating Care and Collaborating with Other HCPs .............67

Sub-theme: Being Accountable for Professional Scope and Role ...............68

Theme four: Wading Through the System and Working Through the Process ...........71

Sub-theme: Coping with Unnecessary Technological Complexity ..............72

Sub-theme: Normalizing Workarounds .................................75

Theme five: Learning and Unlearning Over Time ............................... 77

Sub-theme: Adapting to the Learning Curve ................................78

Sub-theme: Meeting the Learning Needs of NPs .......................... 80

Summary of the Findings .............................................. 84

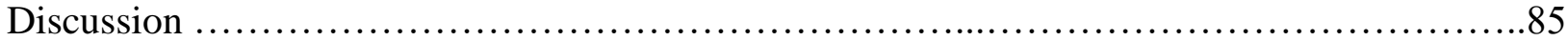

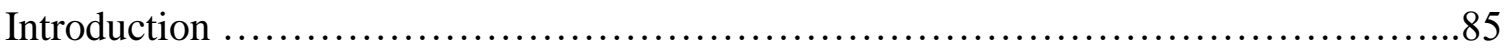

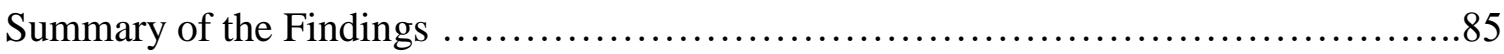

Methodological Considerations.............................................. 86

Insights into NP eMedRec Use ............................................ 87

The NP Role and eMedRec ............................................. 88

Implications for Practice ......................................91

Implications for Policy and Administration ..........................92

Implications for Education ...................................93

Understanding the Pediatric Patient and Family ............................ 94

Implications for Practice ...................................... 95

Implications for Policy and Administration ........................96

Implications for Education........................................97

The Patient Safety Lens ............................................. 98

Implications for Practice........................................ 101

Implications for Policy and Administration .........................102

Study Limitations......................................................... 103

Future Research ......................................................... 104

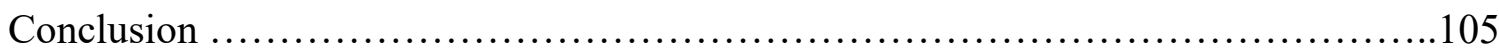

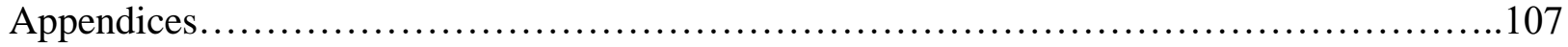




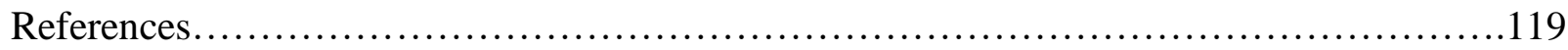




\section{List of Figures}

Figure 1 The Lived Experience of NP eMedRec..............................51

Figure 2 Caring for the Patient and Family.................................52

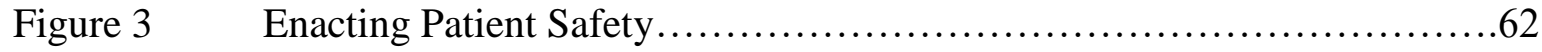

Figure 4 Practicing with the Professional Role and Scope.......................67

Figure $5 \quad$ Wading Through the System and Working Through the Process...........72

Figure $6 \quad$ Learning and Unlearning Over Time............................... 78 


\section{List of Appendices}

Appendix A:

Ryerson REB

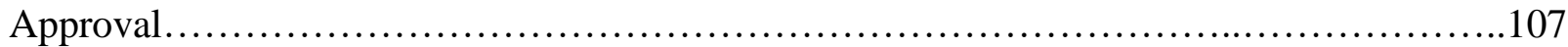

Appendix B:

Study Site University REB Approval............................................109

Appendix C:

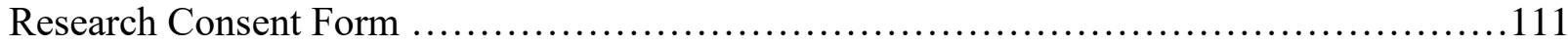

Appendix D:

Email Recruitment Letter .................................................... 116

Appendix E:

Interview Guide..................................................................... 117 


\section{Chapter 1 - Introduction}

Technology is ubiquitous in the lives of many in Western culture, yet despite this trend, there is much to be understood regarding the use of technology in healthcare. Healthcare is a complex industry charged with the task of conducting mission-critical work which ultimately affects the lives of patients and their families. An understanding of technology use is particularly needed in pediatric acute care environments due to the vulnerability of children to medication and other errors. A phenomenological study was undertaken to examine the experiences of professionals who use computerized systems intended to promote patient safety. The report of this study includes five chapters: 1) Background and Significance, 2) Literature Review, 3) Methodology, 4) Findings and 5) Discussion. In this chapter, background information about the research topic and the context for the study are provided. The research problem and question are introduced at the conclusion of the chapter.

\section{Background \& Significance}

Patient safety has become an integral topic within today's healthcare organizations. It has been of particular interest since the Institute of Medicine (1999) published a report entitled To Err is Human in which the prevalence of preventable medical errors was brought to the forefront and challenged healthcare professionals (HCP) and administrators to reflect on the level of risk associated with the services they provide to their patients. Almost a decade later, the World Health Organization [WHO] (2008) noted that adverse medication incidents are among the most common forms of patient safety concerns within developed nations. Many of these patient safety concerns related to medications are often a result of the provider having insufficient information about the patient to make appropriate clinical judgments (WHO, 2008). 
To address these concerns, the Institute for Safe Medication Practices (ISMP) Canada (2008) focused on Medication Reconciliation (MedRec) as one of strategies that can contribute to improved medication safety for patients. MedRec can be a manual, paper-based process or it can be electronic and it aims to address the issue of "....assuring medication accuracy at transitions in care" (ISMP Canada, n.d.). Transitions in patient care take place at points in which patients move from one part of the health care system to another such as from their home in the community to an acute care inpatient facility. Transition points can also take place within the same facility such as a patient transfer from an intensive care unit (ICU) to an inpatient medical unit. Finally, transitions can also refer to a change in the responsible medical team in which there is a transfer of services. It is well documented in the literature that handover between HCPs and points at which patients transition between healthcare services, increase the risk for medication and other preventable errors (Manias, Gerdtz, Weiland and Collins, 2009; Manser and Foster, 2011; Thomas, Schultz, Hannaford and Runciman, 2013). As a result, MedRec offers a moment for the healthcare team to reflect on their patient's current medications and plan of care in order to make informed decisions about future medication management.

The key components of MedRec include obtaining the best possible medication history (BPMH) from the patient/family in addition to a secondary source such as a pharmacy medication list or medication bottle (ISMP Canada, n.d.). Based on the BPMH, the patient's existing medications are reconciled with the new inpatient medication orders. The reconciliation process involves having the prescriber review each medication and make a decision about ordering or discontinuing these medications based on the admission diagnosis and plan of care (ISMP, 2008). At a large, urban pediatric hospital, MedRec was initially implemented in 2008 
as a paper-based process in which the nurse or pharmacist was responsible for obtaining the BPMH and the nurse practitioner (NP) or physician (MD) would complete the reconciliation.

In 2014, ISMP Canada in collaboration with Canada Health Infoway, a governmentfunded eHealth-focused organization, published a toolkit for the implementation of electronic MedRec. Electronic Medication Reconciliation (eMedRec) aims to digitize a previously paperbased process in order to ensure consistency of patient medication management across the continuum of care (The Electronic Medication Reconciliation Group, 2014). This toolkit was designed to facilitate the implementation and adoption of eMedRec and help organizations transition from a paper-based to an electronic process. It is important to note that this Paper to Electronic MedRec Implementation Toolkit was published in September of 2014 while the implementation process at the study site began in April 2014, prior to the document's publication date.

Within the organization of focus for this study, eMedRec was implemented based on a recommendation from Accreditation Canada. It is also important to note that leaders responsible for MedRec at the study site reported that there were significant challenges with the adoption of the paper-based eMedRec process due to the complexities of clinician workflow. For example, the MedRec form remained in the patient's chart at the front desk and because the documentation was hand-written, medication information was often difficult to read. Also, the fact that MedRec was not electronic meant that it was kept separate from the order entry process so a prescriber would need to be in the same physical location as the patient's chart to review the home medication list on the form. These challenges with the paper-based process, among others, also supported the claims that an electronic version would be easier for clinicians to adopt due to its connection to the existing computerized provider order entry (CPOE) system. In this way, the 
implementation of eMedRec was deemed to be the next step in the process to ensure improved patient safety as patients transitioned into the hospital and between different departments once admitted.

The integration of technology such as CPOE and eMedRec into clinical practice has rapidly become the norm for many healthcare organizations. In the current technology driven, information age, professionals and administrators are challenged to keep pace with the rapid development of new technologies in order to safely and efficiently deliver patient care (Harrington et al., 2011; Xiao et al., 2014). Over the last two decades, scholars in the field of health informatics and patient safety have expressed concerns regarding the prevalence of medical errors and the use of healthcare technology has been cited as a potential mitigating factor (Feng, Bobay \&Weiss, 2008; Karsh, Holden, Alper \& Or, 2006). For example, Karsh et al., (2006) discuss a human factors paradigm, in which systems are designed for HCP performance and can therefore mitigate medical errors via improved technology and process design. In fact, Bates and Gawande (2003) suggest that the key steps to improving medication safety, in particular, include systems that “...can improve communication, make knowledge more readily accessible, require key pieces of information... assist with calculations, perform checks in real time... and provide decision support” (p. 2526). These information and communications technology (ICT) functions recommended by Bates and Gawande (2003) parallel the recommendation for eMedRec implementation noted by ISMP Canada (n.d.) and Accreditation Canada.

ICT, such as eMedRec, have the potential to decrease risks associated with traditional methods of patient medication management because there are built in clinical decision support functions and dose checking features. With the prevalence of such highly developed technology 
within our society, the risk of medication errors should be decreased, in theory, via the introduction of technology in patient care environments (Bates \& Gawande, 2003). Therefore, eMedRec should be able to address many of the concerns regarding points of transition in patient care because there are flags within the system to notify clinicians when MedRec is complete/incomplete or missing information. The allergy, duplicate order and dosing alerts within the CPOE module should also address some of the risks to patient safety. Because eMedRec is linked to the CPOE system within the electronic health record (EHR), there is a level of integration between different aspects of the computer systems that should provide some assurance that there are minimal gaps in the medication management process. For example, once the patient's home medications are entered into the system, the prescriber does not need to reenter the medication into the CPOE section of the system. Instead they can continue the home medication as an inpatient order with a few clicks of the mouse. This integration between the study site's eMedRec and CPOE components is an example of how technology can be used as a method for increasing patient safety.

Although the need to increase patient safety may be used justify the implementation of health information technology (IT), there is a lack of consistent research evidence demonstrating an increase in safety and decrease in risk (Campbell et al., 2006; Canada Health Infoway, 2008; Del Beccaro, Jeffries, Eisenberg and Harry, 2006; Han et al., 2005; King et al., 2003). For example, there have been studies finding an increase in mortality after technology implementation, no change in mortality and others found a decrease in medication errors (Del Beccaro, Jeffries, Eisenberg and Harry, 2006; Han et al., 2005; King et al., 2003). To add further complexity to the evidence surrounding this topic, Campbell et al. (2006) noted a decrease in medication errors that resulted in patient harm but also saw the emergence of new, unexpected 
errors such as selecting the wrong item from a drop-down list or typing information into a miscellaneous field which negates any clinical decision support features. In fact, many of the studies that focused on establishing the impact of components of the EHR were conducted over 10 years ago. It is also possible that negative results may not be published.

Canada Health Infoway (2008) noted that the outcomes-related evidence pertaining to eMedRec and similar eHealth technology is inconclusive. King et al (2003) conducted a study to demonstrate the impact of CPOE on mortality rates in a pediatric ICU but, upon closer consideration, there were several other factors that may have impacted the mortality rates such as the patient diagnoses at the time of the study and the CPOE implementation process. When similar studies demonstrating a positive or negative impact on patient safety were examined closely, there were extraneous variables that are difficult to control. For example, Schnipper et al. (2009) demonstrated a decrease in potential adverse events because of eMedRec implementation. This study was a randomized control trial (RCT) and as such, not entirely reflective of the dynamic clinical realities that exist in a hospital, especially in pediatrics where the family is included as the recipient of care with the patient. Schnipper et al. noted a $28 \%$ decreased risk for unintended medication discrepancies with a potential for harm after the implementation of an eMedRec system [intervention group = 170 discrepancies and control group $=230]$. The authors noted however, that there may have been additional factors limiting further gains from the intervention such as patient and provider knowledge of the patient medication history, a lack of software integration between ambulatory and inpatient departments as well as system usability and clinicians' uptake of the new process (Schnipper et al.).

Similarly, within the organization of focus for this study, there may be multiple factors contributing to the success of the eMedRec implementation that cannot be adequately explored 
via experimental research. Many of the studies that sought to measure the impact of technology use on patient safety were conducted in a real life context and often using a retrospective or pretest/post-test design (Del Baccaro et al., 2006; King et al., 2003; Han et al., 2005). Even with a less rigid research design, the results of the aforementioned studies do not address the gap in knowledge related to the effective use of technology in healthcare. Holden et al., (2013) builds on the work of Carayon (2006) among others in the Systems Engineering Initiative for Patient Safety (SEIPS) 2.0 model which depicts the healthcare system as a complex system in which multiple aspects of the system including patients and workers impact the processes and outcomes of the system. While this model described by Holden et al., (2013) highlights the factors that contribute to outcomes such as patient safety and allow for an analysis of an overall system, there remains a need to understand the specific human experiences that contribute to these organizational outcomes. A qualitative approach focusing on the human science aspect of eMedRec use has the potential to reveal not only factors that contribute to the success or failure of healthcare technology use, but also why these factors have an impact on the process.

It has also been observed in practice that a key factor impacting the successful adoption of eMedRec at the study site is the ease with which HCPs can navigate through the software. The desired patient safety benefits of eMedRec may not be realized if the software that was implemented to improve patient safety is not used effectively. The overall eMedRec adherence rate of $76 \%$ for the NP and MD components of eMedRec at one year post implementation at the study site and anecdotal concerns expressed by users of the system present a challenge to understanding the effectiveness of eMedRec. There is evidence to suggest that automation and engineered controls such as those included in eMedRec should contribute to decreased errors and increased patient safety; however, it is difficult to arrive at any conclusions about outcomes if 
practitioners do not use the technology consistently or effectively (Bates and Gawande, 2003;

Karsh et al., 2006). Adoption and usability concerns may be indicators of a gap in knowledge that may be better revealed through qualitative inquiry.

Because of the lack of qualitative research in this area and the discrepancies between the various outcomes-based studies, further research is warranted. From a sociotechnical perspective, Clegg (2000) states that technology is often developed or implemented in the absence of end user input and thus, from a highly technical perspective, results in the need to redesign user workflow to fit the technology. Applied to a healthcare context, redesigning provider workflow to accommodate the technology may cause clinicians to lose sight of the software's fundamental clinical purpose to improve patient safety. Also, a system designed from a highly technical perspective without a good understanding of user needs may contribute to significant usability concerns (Clegg, 2000).

Because of the need to better understand usability issues, there may be challenges in ascertaining the nuances that exist in and give context to the eMedRec process from a human science perspective. Middleton et al., (2013) states that "[t]he ability to perform meaningful, reproducible, objective usability metrics for EHR systems is limited by the socio-technical nature of the systems" (p.e4). In other words, there are other socially dependent factors, which challenge the traditional, technical, objectivity-focused methods of usability testing. Examples of these socially dependent factors are the varying professional roles of each user, the geographical location of the user or patient and the asynchronous nature of data entry and retrieval by HCPs (Middleton et al., 2013). It is possible that a qualitative approach that allows for an in-depth examination of NP experience may be advantageous as other approaches using pre-set variables are unable to capture the nuances of eMedRec use in this context. An understanding of 
clinicians' experience with eMedRec use may contribute to the development of improved, novel approaches to health IT design and implementation.

\section{Study Purpose and Question}

The purpose of this qualitative study was to provide insight into processes related to eMedRec at the study site by understanding and assigning meaning to the use of eMedRec as the lived experience of NP. The research question was thus: what is the lived experience of eMedRec use by NP at a large pediatric acute care hospital? The secondary research question to be addressed was: what are the factors affecting the use of eMedRec by NP.

Using an interpretive lens, the philosophical foundation of this study was Heideggerian hermeneutic phenomenology. The work of Van Manen (1990; 2014) informed the methodology and guided the research methods because of its potential to facilitate the description and interpretation of NP eMedRec as a human experience. The study's guiding philosophy and the work of Van Manen $(1990 ; 2014)$ are addressed in greater detail in chapter three. 


\section{Chapter 2 - Literature Review}

\section{Introduction}

This chapter begins with an overview of the search strategy for the literature review. The literature presented is discussed in relation to the themes of CPOE and the outcomes perspective as well as technology usability and the user experience in healthcare. In the final section, a summary of the evidence provides a succinct analysis of the current literature. Gaps in the evidence are identified to support the rationale for this study.

\section{Search Strategy}

The search strategy was conducted in approximately three phases using CINAHL and ProQuest databases. The following key words were used: medication reconciliation, informatics, computerized provider order entry, CPOE, usability, workflow, human factors and workarounds. Each search yielded approximately 50 to 1000 hits. Some key words were entered individually and others in combination using the Boolean operator "AND" with the search refined to peer reviewed content and English language. The timeframe for the initial search was from 2000 to present due to particular advancements in health IT during that period of time. When focusing specifically on electronic medication reconciliation, the time range was narrowed to 2009 to filter irrelevant articles. Approximately 25 abstracts or full-text articles were reviewed and articles were excluded if the topic or search terms were not mentioned in the title or abstract. Articles were also excluded if they were presented from an academic or educational perspective instead of a direct clinical practice context.

\section{CPOE: The Outcomes Perspective}

There has been increased awareness surrounding the issue of patient safety and the need to decrease medical errors nationally and internationally. It is not surprising that the healthcare 
technology literature has historically focused on measuring outcomes of technology implemented in various healthcare practice environments. The following is a summary and critique of those findings.

As a method to reduce the risk of medication errors, CPOE was one of the first types of ICTs implemented at the hospital site used for this eMedRec study. In a study conducted at a Canadian pediatric hospital, King et al., (2003) noted a decrease in the study site's rate of medication errors post CPOE implementation but there was no difference in the rate of actual or potential adverse medication events. King et al. describes medication errors as those made by a practitioner during the course of ordering, dispensing or administering a medication. Adverse medication events (AME) however, are unforeseeable events that cause actual or potential harm to the patient and may or may not be a result of an error (King et al.). The discrepancy between the rates of medication errors and AMEs could be due to methodological limitations of the study such as the use of a passive reporting system that relies on the HCP to report errors (King et al.). Another reason why King et al., may have noted a difference in the medication error rate but no difference in AME rates post CPOE implementation could be that CPOE contributed to the reduction of medication errors while also contributing to new types of errors that were not previously defined. In fact, one study observed that new types of errors constituted $7 \%$ of the total 324 unintended consequences of CPOE (Campbell et al., 2006). Unintended consequences could be one explanation why King et al. found that the medication error rate (MER) decreased, while the AMEs remained the same post CPOE implementation.

Further inconsistencies have been noted in two similar studies regarding $\mathrm{CPOE}$ implementation conducted at pediatric institutions. One facility experienced an unexpected increase in mortality rates while the other did not (Han et al., 2005; Del Beccaro et al., 2006). In 
the first study, Han et al. (2005) observed an increase in pediatric mortality rates after CPOE was implemented in a pediatric intensive care unit (PICU) however the author failed to acknowledge how systemic factors may have skewed the results. Some of these factors included a policy restricting providers' ability to enter electronic orders prior to the patient's arrival as well as technological challenges exclusive of the actual CPOE module (Han et al., 2005). A potentially seasonal increase in patients with multiple comorbidities could have also played a role in the noted increased mortality rate post CPOE implementation.

In the second of the two studies regarding CPOE implementation, Del Beccaro et al., (2006) observed that mortality rates were not statistically different post-implementation compared to the pre-implementation phase. Del Beccaro et al., (2006) reviewed the mortality rates of patients admitted to the PICU 13 months before and after CPOE was implemented. In addition, they separately analyzed the data from patients transferred from other facilities in order to discuss the unique characteristics of that population and noted that they may have had an inherently increased risk of mortality compared to that of other PICU patients (Del Beccaro et al., 2006). This detail is noteworthy because the entire sample from Han et al., (2005) consisted of external patient transfers to their PICU which could mean that, based on the observations noted by Del Beccaro et al., (2006), the sample of the former study may have already been at a high risk for mortality thereby skewing the CPOE outcomes. Conversely, Del Beccaro et al., (2006) noted that their pre-implementation sample of patients had a higher level of acuity than those in the post-implementation group. This difference in patient acuity could explain why the results of this study were not statistically different because the decrease in mortality rates noted immediately post implementation could likely be attributed to the overall health status of the patients in either cohort. 
The differences between the results of the studies published by King et al., (2003), Del Beccaro et al., (2006) and Han et al., (2005) reveal the complex nature of CPOE and technology for the provision of patient care in a pediatric tertiary care setting. The eMedRec system at the site for the current study incorporates CPOE as a portion of the medication reconciliation process. Therefore, the studies cited here involving CPOE are highly relevant because the gaps in knowledge regarding variations in patient-related outcomes may be addressed via qualitative inquiry and phenomenology in particular. As a qualitative researcher exploring the phenomenon of eMedRec use, the acknowledgement of the extraneous variables evident in the aforementioned studies provides the basis for exploring the contextual factors associated with the use of similar technology in a complex healthcare setting.

\section{Technology Usability and User Experience in Healthcare}

Usability for the purposes of this study is conceptualized as the ease with which a user can navigate the human-computer interface. User Experience (UX) has varied definitions in the literature but the definition by Hassenzahl and Tractinsky (2006) was used for this study. These authors propose that UX is a "...a consequence of a user's internal state..., the characteristics of the designed system..., and the context within which the interaction occurs" (p. 95). Software usability concerns have emerged in the literature as an aspect of healthcare technology that should be considered to address the inconsistencies in outcomes-based eHealth research (Canada Health Infoway, 2012; Keohane et al., 2008; Xiao et al., 2014). To address these inconsistencies, recent studies have explored EHR usability and HCP workflow. The following paragraphs provide an overview and critique of these studies.

Based on time in motion studies and user perception, there is evidence supporting the notion that efficiency either decreases or remains the same when an EHR is implemented 
(Carrington and Effken, 2011; Asaro and Boxerman, 2008; Ward et al, 2011). In a study evaluating nurses' perceptions of the strengths and limitations of an EHR for documenting significant patient events, Carrington and Effken (2011) reported that nurses from three different sites felt that their EHR decreased the efficiency of HCP communication. eMedRec is an aspect of the patient's EHR at the site for this study and by way of the medication history, known as the Outpatient Medication Record, eMedRec is also a vehicle for interprofessional communication about the patient's medications.

Ward et al. (2011) also examined nurses' perceptions of an EHR using a pre and postimplementation design. Nurses in this study were positive overall about the technology preimplementation with minimal change in this perception after training; however, there was a significant decrease in their positive scores after implementation. In particular, nurses' confidence in interprofessional communication, patient safety and the quality of patient data decreased compared to their pre-implementation perceptions (Ward et al., 2011). The results may suggest that there were concerns about the technology but the nurses' pre-implementation expectations, compared to the reality they experienced once the technology was implemented, could have played a major role in the post-implementation results. In other words, there may have been a cognitive or emotional aspect of the nurses' experience that affected their expectations and thus disappointment in the technology post implementation. This gap in understanding the way in which the nurses in experienced the use of the newly implemented technology could be addressed by engaging in semi-structured interviews with the participants. In this way, it may have been possible to explain their decreased confidence in the features of the technology. It is possible that this understanding could be obtained via a deeper conversation aimed at describing and understanding the meaning behind their responses. 
Another study that focused on user experience examined physicians' perceptions of an integrated EHR before and after implementation in an outpatient setting (Vishwanath, Singh \& Winkelstein, 2010). The authors found that physicians' prior expectations of the technology impacted their post implementation perceptions which were based less on objective evaluation of the system and more on personal beliefs of what the application should be able to accomplish (Vishwanath et al., 2010). The authors also noted that the learning curve that was introduced as a result of the new EHR had minimal impact on the physicians' perceptions of the technology (Vishwanath). The study described by Vishwanath et al. allows for an understanding of user perceptions and expectations of technology as well as how such perceptions can influence the adoption of technology. Vishwanath et al. and the aforementioned studies however, discuss the notion of perception versus an actual account of the participants' experiences. While, Vishwanath et al. provide insights into how user perceptions change or remain the same over time, there remains a gap in understanding why the physicians' perceptions of the technology do not change. It is possible that a methodology allowing the users of the system to describe their experiences and interpret their perceptions might illuminate other aspects of this phenomenon but at a deeper level of analysis and understanding.

Carrington and Effken (2011), explored nurses' perceptions of EHRs, however, they did not provide an understanding of why these nurses perceived the EHR as inefficient. Ward et al., (2011) also conducted a quantitative evaluation of nurses' experiences with technology, but their study focused on the impact of the implementation of technology and how it changed the workflow of the documentation process. While each study provides valuable knowledge for the understanding of how HCPs perceive the use of technology in healthcare, neither study explored why the HCP may feel that the technology is inefficient, why they may have high expectations of 
this technology, and why it fails to meet their expectations. A phenomenological exploration of the health professional's experience using a particular technology could provide an understanding of the tacit realities of human computer interactions and the related contextual factors specific to patient care.

Collins et al., (2012) and Keenan et al., (2013) explored the ease with which nurses and the interprofessional team document and therefore access pertinent patient information (Collins et al., 2012; Keenan et al., 2013). Keenan et al. utilized ethnographic observation to study nursing and interprofessional communication in addition to the workarounds that occurred when the EHR failed to support their workflow. While this group of authors aimed to describe the process of communicating patient information, workarounds such as the reliance on paper in the presence of an EHR were identified (Keenan et al.). Collins et al. (2012) noted that fragmented electronic flow sheets and medication administration records, for example, did not allow for the communication of pertinent clinical information that "...belong[ed] together..." (p. 96). This creates extra steps for nurses to document significant events and has a potential impact on patient safety as HCPs may miss items that provide them with a complete clinical picture of their patient. Similarly, eMedRec is set up into three different work spaces within the application; therefore, participants in a phenomenological study regarding eMedRec use may identify any fragmentation in the application in their description and interpretation of their experience.

The findings of these studies suggest that EHRs need to employ a design in which the ways the user interacts with the technology are considered thoughtfully and made a priority while also ensuring that extensive usability testing occurs prior to implementation (Collins et al., 2012; Flanagan et al., 2013; Harrington et al., 2011; Keenan et al., 2013). Because this eMedRec 
study is focused on the experience of HCPs using a particular software, the interview data may bring to awareness the usability concerns that may not have been addressed in the past.

\section{Theories of Human-Computer Interaction and the Complex System}

The impetus for exploring the phenomenon of eMedRec from a qualitative perspective is a result of the low percentage of adherence to eMedRec use at the study site one year postimplementation. Having this contextual knowledge, an overview of the technology literature is provided to facilitate an understanding of the existing theories surrounding this phenomenon. The following section includes a discussion of the Technology Acceptance Model (TAM) and UX as noted in recent literature.

\section{User Experience (UX)}

There is an increased emphasis on the need for consideration of usability and user experience related to healthcare technology during implementation. In an article advocating for increased research efforts in the area of UX, Hassenzahl and Tractinsky (2006) argue that there has been a necessary shift from a task-related focus in technology usability research to a more experiential one. In the field of human-computer interaction (HCI), researchers primarily focused on "...the achievement of behavioural goals in work settings" (Hassenzahl and Tractinsky, 2006, p.92). In other words, information technology professionals and scholars assumed that the primary factor to consider was task completion and the functional aspect of the application; however, there are many other aspects of human computer interactions that should be considered such as the affective and esthetic.

Hassenzahl and Tractinsky (2006) propose a model of UX that articulates the concept as a humanistic one in which there are three converging perspectives that contribute to an overall understanding of UX. The authors indicate that UX should consider factors beyond the 
instrumental tasks and should incorporate an understanding of the emotion and affect of the user in addition to the complex, contextually-bound nature of the actual experience of using the technology (Hassenzahl and Tractinsky, 2006). This understanding of UX that goes beyond the quantification of tasks to be completed, lends well to the focus of this study because phenomenology emphasizes the temporal, contextual experience of the phenomenon of study as articulated by the participants. While Hassenzahl and Tractinsky (2006) discuss technology that is not situated in a healthcare context, the principles they address regarding the significance of the subjective, aesthetic aspects of human-computer interactions are philosophically congruent with interpretive phenomenology.

In a UX study, the researchers observed users' evaluation of an arts-based technology in the moment of their actual experience and at the end of the experience (Hassenzahl \& Ullrich, 2007). Instructions were provided to one cohort of the study so that they had goals to achieve during their use of the technology while the other cohort was not given instructions and therefore no goals were stated. Hassenzahl and Ullrich found that the goal-oriented participants displayed a higher level of mental effort when they encountered standard usability concerns within the technology compared to the control group and "...mental effort was negatively related to affect..." with both factors influencing the participants' evaluation of the technology (p.435). Otherwise stated, as mental effort increased, participants' favourable evaluation of the technology decreased. While not specific to healthcare technology, the authors uncovered an interesting cognitive aspect of UX that could explain why a HCP might have strong negative feelings towards a complex technology required for complex tasks. 


\section{Technology Acceptance}

In a large tertiary care centre, technology is used for various aspects of patient care delivery. The successful adoption of technology is dependent on many contributing factors. The Technology Acceptance Model (TAM) is one way of understanding IT users' intention to use the newly implemented technology. Holden and Karsh (2010) conducted an extensive review of the

literature and provided an overview of the theory's utility for healthcare. The key components of TAM are perceived usefulness (PU) and perceived ease of use (PEOU) of the technology, which influences the user's attitude and behavioural intention to use the technology (Holden \& Karsh, 2010). The authors defined PU as the use of health IT that improved the user's work performance or enhanced their work in some way while PEOU was vaguely defined as the lack of mental or physical effort required to complete the task (Holden \& Karsh, 2010). The main critique of this theory is that technology utility and task completion is given too great a focus versus the people using the technology. In a complex pediatric acute care environment, there are many people and tasks that interact to provide patient care and that humanistic experience might be missing when a phenomenon is viewed solely from this lens.

This understanding of TAM, however, complements the earlier discussion regarding UX because the common thread between the two theories is that mental effort and task completion seem to be central to how an IT user experiences and adopts new technology. UX, as defined by the studies examined in this literature review, reveal an understanding of the cognitive processes inherent in human-computer interactions that are beyond the basic fulfillment of roles or tasks for a job and as such provides a complementary perspective from which to explore the phenomenon of eMedRec use. 


\section{The Complex System}

Holden, Carayon and Gurses, (2013) added to the existing Systems Engineering Initiative for Patient Safety (SEIPS) to create SEIPS 2.0. This model involves the structures of the work system and how they interact to affect the related processes and the ultimate outcome of the system (Holden, et al.). The key feature or addition to SEIPS 2.0 is the inclusion of the patient in the role of person in the work system. This update to the original model is reflective of the increased understanding of the role that patients play in the functioning of the complex systems within healthcare. Holden et al., (2013) describes the patient as a person who may interact with other people, technologies, and the organizational environment as a whole while using their individual or collaborative efforts to contribute to outcomes for the organization. This model proposes a more robust method of accounting for the way in which patients and HCPs interact with technology to contribute to a desired outcome and thus, has the potential to facilitate an understanding of the context surrounding technology use. However, the SEIPS model does not articulate individual experiences but provides a method for understanding those experiences; therefore, the gap in knowledge in this context is the lack of understanding of the individual experience of those using technology such as CPOE or eMedRec.

\section{Phenomenology in Healthcare Technology}

There are many phenomenological studies in nursing and a small body of the same in information technology; however, few phenomenological studies have been used to understand HIT. The following three studies used phenomenology as the methodology of choice.

The first study explored the experiences of radiology professionals engaged in the process of computerized tomography (CT) and magnetic resonance imaging (MRI). Lundvall, Abrandt-Dahlgren and Wirell (2014) explored the work processes of radiographers with a 
specific focus on their roles and responsibilities. The aim of the study was to explore the “...general tasks and responsibilities of their work", yet there is no mention of radiographers' lived experience in the aim or research question (Lundvall, Abrandt-Dahlgren and Wirell, 2014, p.49). The authors employed interpretive phenomenology by conducting interviews and direct observations for data collection (Lundvall et al.). The authors found that radiographers framed their experience in terms of their work with the patient and adjusted the technology and radiography process to suit the needs of the patient (Lundvall et al.). The themes of Planning the examination, Producing the images and Evaluating the examination helped to convey the lived experience of the participants (Lundvall et al.).

For this study there were methodological inconsistencies and a philosophical incongruence between their data analysis and the stated methodology. For example, the first author employed bracketing, whereby their experience as a radiographer was set aside in order to conduct this study (Lundvall et al., 2014). While this approach may have been appropriate for Lundvall et al., it is not consistent with Van Manen's approach and, therefore, not suitable to employ in this eMedRec study. In fact, Heidegger emphasizes that the researcher cannot completely separate their experiences from the phenomenon of study and those experiences in fact contribute to the co-construction of meaning between the researcher and the participants (Dowling, 2007; Van Manen, 1990; Van Manen, 2014). It is difficult to evaluate the rigour of the study by Lundvall et al. without additional information about the philosophical approach employed by the authors. While Lundvall et al. achieved their aim of learning about the roles and responsibilities of radiographers in CT and MRI, their application of phenomenology to this study brings into question the themes arrived at during the course of their study. 
The second study explored the experience of nurses caring for patients supported by technology in an intensive care environment. The study aim was to "...describe the meaning of the experience of Thai nurses caring for persons with life-sustaining technologies..." and the authors employed a qualitative approach that was informed by hermeneutic phenomenology (Kongsuwan \& Locsin, 2011). Kongsuwan and Locsin employed Van Manen's (1990) interpretive phenomenology and revealed that the participants valued competence in terms of caring for the patient regardless of their level of technological competence. Kongsuwan and Locsin conveyed their findings via rich, detailed descriptions of the participants' experiences however their criteria for establishing rigour was based on the notions of credibility, transferability, dependability and confirmability which has been critiqued over the last few decades due to its positivist leanings (Sandelwoski, 1986;1993). Perhaps an alternative method of establishing rigour could have been employed to ensure congruency with the authors' stated methodology. In the case of Kongsuwan and Locsin (2011), phenomenology as a methodology was applied to the data analysis however the authors did not make clear their philosophical foundations throughout the article.

The third study for review also examines the experience of critical care nurses but is very specific to the management of critical technology for patient care. Alasad (2002) employed Heidegger's hermeneutic phenomenology and addresses its philosophy guiding the methods of the study. The author continues to discuss the guiding philosophy throughout the text and provides a method for evaluating the study's rigour that is congruent with the methodology and philosophical foundation. By employing Van Manen's (1990) thematic analysis, the themes of "Safe and in control", "Being technically competent" and "Demanding and time consuming" emerged from the data (Alasad, 2002, p. 409-410). These themes parallel comments stated by 
colleagues at the study site about eMedRec in that it is used to provide a level of patient safety and there are challenges with the technology which result in the perception that the use of eMedRec is demanding and time consuming. The phenomenon of eMedRec use however explores the use of a technology that is not physically connected to the patient and functions as clinical documentation as well as CPOE and clinical decision support.

\section{The Nurse Practitioner and Patient Experience}

Although the technology has a significant presence in the lives of those involved in healthcare, the patients and their HCPs are the people for whom these technologies are developed and it is important to understand the human perspective when exploring the phenomenon of technology use. Holden et al., (2013) discussed the need to understand the patient's involvement in the functioning of a complex system. Higgins, Larson and Schnall, (2017) explored the concept of patient engagement as they found that it was a term often used in healthcare but never defined. The concept analysis provided insights into what patient engagement means for the nursing discipline. Higgins et al. can also provide an understanding of what it means to engage with patients in their care because the primary role of the NPs in this study is direct patient care.

In addition to the patient, the NP plays a significant role in the complex system of an acute care pediatric hospital. Rashotte and Jensen (2010), conducted a hermeneutic phenomenology of the NP role and found that it was a transformational journey encompassing the themes of Being Called to be More, Being Adrift, Being an Acute-Care NP, Being Pulled to be More and Being More. Overall NPs in this study expressed contradictory feelings of insecurity and confidence in their expertise depending on their level of experience (Rashotte and Jensen, 2010). They also shared the challenge of being caught between the role of a Registered 
Nurse and MD; a sub-theme that Rashotte and Jensen (2010) referred to as Being a Wearer of Two Hats. This study is an important one as it brings to light the complex realities of the NP experience. With regards to the study of NP's experiences using eMedRec, Rashotte and Jensen (2010) offer an understanding of the NP role and the utility of hermeneutic phenomenology to uncover the implicit aspects of the NP experience.

Continuing an exploration of the NP role, LeGrow, Hubley and McAllister (2010) present a conceptual framework for Advanced Practice Nursing at a large pediatric acute care facility. This conceptual model was defined by the authors after determining that the existing framework was inadequate in its ability to capture the full scope of the pediatric advanced practice nurse. Thus, a humanistic framework was developed encompassing other frameworks as its structure, a description of the work process including the child and family at the centre and the outcomes which include the enhancement of child and family health and the advancement of pediatric nursing practice (LeGrow et al.). This conceptual framework, while continuously evolving, is relevant for this study of NPs' experiences using eMedRec because it illuminates insights into the NP role that are specific to a pediatric acute care setting.

\section{Summary of the Evidence}

In an attempt to address concerns surrounding the use of technology for patient care, many studies included in this review employed a quantitative research design with a particular focus on outcomes related to patient care or practitioner workflow. When the research emphasis is directed towards patient outcomes, the independent variables tend to be expressed in terms of rates, and in the case of medication errors, they often rely on passive, self-report systems (Del Beccaro et al., 2006; Han et al., 2005; King et al., 2003). When provider workflow is the chief concern, time in motion studies and surveys have been used for quantitative analysis and direct 
observation or ethnographic methods have been employed from a qualitative perspective (Asaro and Boxerman, 2008; Carrington and Effken, 2011; Collins et al., 2012; Keenan et al., 2013; Vishwanath et al., 2010; Ward et al, 2011).

The common recommendation from the outcomes focused research is the need for usability testing and an increased emphasis on UX. The technology literature focuses on the cognitive and emotional aspect of UX with a correlation between mental effort and user affect (Hassenzahl and Tractinsky, 2006; Hassenzahl and Ullrich, 2007). While there might be a perceived tension between the need to understand the instrumental goals of the IT user compared to the modern discourse of aesthetics and affect, both perspectives can provide an understanding of UX related to health information systems such as eMedRec.

There are limited studies involving health information systems using a phenomenological approach and of those studies, the methodology is inconsistently applied. There is a significant lack of qualitative research that explores components of the EHR such as CPOE and eMedRec, The study of human-computer interaction via qualitative inquiry has the potential to uncover implicit meanings associated with these constructs. As a result, the current study addressed this gap in knowledge by exploring the human-computer interaction and its varied contexts via the lived experiences of NPs who use the computer system regularly. 


\section{Chapter 3 - Methodology \\ Introduction}

The use of healthcare technology has been minimally explored in the literature from a qualitative perspective and even fewer have employed a phenomenological approach. eMedRec use can be understood by employing a phenomenological approach to uncover the lived experience of an individual (Van Manen, 1990; 2014). Furthermore, Van Manen (1990; 2014) indicates that an outcome of phenomenological inquiry is assigning meaning to the experience of the phenomenon. This is relevant for the study of eMedRec use among NPs because assigning meaning to the experience of using this mandatory technology may reveal unknown aspects of the human-computer interaction as well as its associated contexts. In this chapter is a discussion of phenomenology, the rationale for selecting interpretive phenomenology and the methods applied in this study including sampling, data collection and analysis. Methods for establishing rigour and ethical considerations are also presented.

\section{Overview of Phenomenology}

Phenomenology is a human science that is both a philosophy and methodology (Dowling 2007; Van Manen, 1990). Van Manen (1990) suggests that human science is the study of human beings and how they exist in and make meaning of their world. Natural science on the other hand aims to study “...objects of nature, things, natural events and the way objects behave" (Van Manen, 1990, p. 3). While the technology is an aspect of the phenomenon under investigation in this study, the primary focus is the human experience of those HCPs who use the technology. Van Manen (2007) refers to phenomenology as a process of attentively reflecting on the "...lived experience of human existence..." (p.12). In this study, the experience of human existence refers to how NPs embody their professional roles when using eMedRec within the context of a 
complex pediatric healthcare system. From a methodology perspective, phenomenology guided by the work of Van Manen $(1990 ; 2014)$ allowed the participants and I to co-construct an understanding of the use of eMedRec via in-depth, conversational interviews. It is important to note that the way in which phenomenology is methodologically executed is directly related to its philosophical underpinnings. As such, the fundamental knowledge development in phenomenology is described in the following paragraphs including why an interpretive, hermeneutic approach guided by the work of Van Manen (1990; 2014) is well suited for this study.

\section{Husserl's Phenomenology}

Considered one of the founding scholars of phenomenology, Edmund Husserl's philosophy is grounded in the post-positivist paradigm in which the pursuit of objective truth is paramount (Dowling, 2007). Husserl's work is sometimes referred to as transcendental phenomenology due to its focus on epistemology, which seeks to uncover the nature of knowledge (Van Manen, 2014). Husserl also believed that fundamental knowledge can be attained most accurately via the lived experience and that all phenomena can be reduced to its fundamental, pre-cognitive state (Dowling, 2007). This pre-cognitive or pre-reflective state would allow the essence of the phenomenon to be unencumbered by researcher interpretation. The phenomenological essence is therefore the reduced, concrete account of the experience prior to reflection, interpretation or understanding (Dowling, 2007; Van Manen, 1990; 2014). To achieve this pre-reflective understanding of a phenomenon, Husserl described the practice of bracketing in which the researcher would suspend or put aside their own experiences to avoid the interpretation of data based on the researcher's experience and to arrive at the objective truth (Dowling, 2007; Van Manen, 2014). In fact, proponents of Husserl's phenomenology believe 
that bracketing is the fundamental aspect of phenomenological research (Dowling, 2007). While Husserl's work provides a basis for human science inquiry focused on the lived experience, the major challenges with his approach, for the purposes of this study, is the notion of bracketing. The focus on description of the phenomenon in the absence of interpretation and the reductionist approach to participants' complex experiences may prove to be problematic given that the researcher is intimately acquainted with the phenomenon of interest.

\section{Heidegger's Phenomenology}

Building upon the work of Husserl, Martin Heidegger, a German philosopher, challenged the prevailing assumption that an objective truth can be arrived at in the absence of its context (Dowling, 2007; Van Manen, 1990). While still aiming to ascertain the lived experience of individuals, Heidegger's work was founded in an interpretive, hermeneutic paradigm and focused on ontology, or the nature and meaning of being. Heidegger's hermeneutic perspective contrasted that of Husserl. This existential view of the lived experience was articulated in Heidegger's (1927) Being and Time publication in which he stated that human consciousness cannot be separate from its existence. Heidegger believed that the way in which human beings experience a phenomenon cannot be separated from their conceptualization of it and therefore, refined the process of bracketing by creating the hermeneutic circle (Dowling, 2007). The hermeneutic circle aims to demonstrate the reciprocal nature of the parts of the text to the whole experience and the symbiotic relationship between description and interpretation in hermeneutic phenomenology (Van Manen, 2014). In this way, hermeneutic phenomenology acknowledges that an understanding of the nature of being requires a description and interpretation of the phenomenon by both the researcher and participants resulting in the co-creation of meaning assigned to the phenomenon. In terms of eMedRec use by NPs at the study site, an interpretive 
approach provides an opportunity for the participants to describe and assign meaning to the nuanced, contextual aspects of this phenomenon.

\section{Van Manen (1990; 2014)}

The work of Max Van Manen has foundations in hermeneutic phenomenology as noted by Heidegger with the majority of his work situated in the area of pedagogy. Van Manen's work has gained popularity among nursing researchers due to his use of the four existentials of temporality, spaciality, corporeality and relationality as methods of description and interpretation of the lived experience (Dowling, 2007). The four existentials are described in further detail later in the data analysis section, however, it is important to note at this point that the existentials are concerned with the concepts of time, space, body and relationship as lived and experienced by the participants. Furthermore, phenomenology employed as a method, according to Van Manen (1990; 2014), allows the participants the opportunity to describe their experience in concrete terms while also interpreting that experience to derive meaning from it.

It is this combination of Husserl's descriptive phenomenology and Heidegger's acknowledgement of human context in their experiences that greatly appeals to me for this study. I fundamentally believe that knowledge and all human experience is inextricably linked to its context and as such, the post-positivist view of Husserl's phenomenology seems inadequate to explore the complexities of technology use in a dynamic healthcare organization. As a Registered Nurse who has experienced the phenomenon, I am unable to suspend my knowledge of eMedRec and the experiences I have gained in order to conduct this study. An interpretive phenomenology guided by Van Manen $(1990 ; 2014)$ however, allowed me to clearly articulate my experiences and positionality in the context of this study while pursuing an understanding of the stated research question. In fact, Van Manen (1990) suggests that the act of proposing a 
research question is inherently interpretive because this question is based on the researcher's experience and interests. In addition to philosophical congruence, Van Manen (1990; 2014) offers a pragmatic approach to hermeneutic phenomenology that allows greater feasibility for a novice researcher but also a method for conveying the findings in an accessible format for research consumers who may be unfamiliar with the highly abstract, philosophical nature of phenomenological research.

\section{Sampling}

\section{Setting}

The study took place at a large, urban pediatric hospital selected due to my experience with the phenomenon at this organization as well as the ease with which I could gain access to the participants. Within this setting, eMedRec is used by Registered Nurses, Nurse Practitioners, Physicians and Pharmacists. There are three components to eMedRec: the outpatient medication record (OMR), the orders reconciliation manager (ORM) and the prescription writer. The first step of eMedRec includes obtaining the best possible medication history (BPMH) from the patient/family and a secondary source such as a pharmacy medication list or medication bottle (ISMP Canada, n.d.). The patient's medications taken at home or another institution prior to the current admission are recorded in the OMR by any of the previously mentioned professionals. It is the responsibility of the nurse and pharmacist however, to confirm and document that a BPMH has taken place.

The medications are then reconciled with the new inpatient medication orders by the nurse practitioner or physician. The reconciliation process involves having the prescriber (NP or MD) review each medication, and make a decision about ordering or discontinuing these medications based on the diagnosis and plan of care (ISMP, 2008). The transfer process is 
similar except the MD or NP reconciles patient orders between the patient's sending and receiving departments. For discharges, the $\mathrm{NP}$ or MD ensures that the inpatient medications are reconciled with the OMR and the discharge prescription to ensure that all medications required at home have been prescribed.

\section{Gaining Access}

Because medication reconciliation is a patient safety initiative and a clinical technology, the directors of quality improvement and patient safety as well as clinical informatics and technology were contacted for approval to implement the study. They advised that consultation with the eMedRec project team members was important; therefore, extensive discussions were conducted with the medical director of quality management, the pharmacy project lead and the medication reconciliation quality analyst. These leaders understood the feasibility of conducting eMedRec research at this organization and agreed that this study would be beneficial.

\section{Sample}

Denzin and Lincoln (2011) question whether it is possible to truly determine an a priori sample size due to the nature of qualitative research. Sandelowski (1995) contends that a sample size needs to be articulated for research planning and ethics board approval purposes. For the purposes of feasibility and in congruence with the methodological approach of this study, a small sample size was employed to ensure that in-depth interviews were conducted with all participants. Morse (2000) also suggests that the sample size of a qualitative study is dependent upon the scope of the study, nature of the topic and quality of the data. This study has a narrow scope with a fairly explicit topic and potentially large volumes of in-depth data from each participant; therefore, a smaller sample size should suffice (Morse, 2000). Although recruitment letters and emails were directed to NPs and MDs, a total of seven NPs responded to the 
recruitment invitation and six were eligible as one did not meet the inclusion criteria because (s)he used eMedRec less than four times per month. No MDs responded to the recruitment letters, emails and posters therefore the total sample size for this study was six NPs. This sample size falls within a commonly cited range; however, data were collected until data redundancy or a theoretical saturation occurred (Sandelowski, 1995). The research question was then changed to reflect the lived experience of NPs instead of NPs and MDs. Otherwise, the study design remained the same.

This study employed purposive and snowball sampling methods. The two recruitment strategies were posters placed in the nursing station of the inpatient units and email recruitment letters. An email was sent to the distribution group for the hospital's Advanced Practice Nurses by the administrative assistant of the Associate Chief of Nursing Practice. The recruitment letter was emailed to physicians via the medical director for quality and safety and the chief residents. Two email notices were sent to NPs approximately two weeks apart to serve as a reminder. The onsite principal investigator for this eMedRec study assisted with recruitment by sending emails directly to individual NPs. Those NPs interested in participating contacted me via email to learn more about the study. If they met the inclusion/exclusion criteria, a copy of the informed consent document was sent for review and they were enrolled in the study.

\section{Sample Population - NP Role in eMedRec}

NPs within the organization are nurses with advanced education, skills and licensure.

They are able order specific diagnostic tests and medications except for controlled substances but they do not have admitting privileges due to organizational policies. NPs are, however, responsible for completing eMedRec. NPs are also able to complete transfer and discharge eMedRec but require an MD signature for a hard copy of prescriptions for controlled substances 
as necessary. While all MDs and NPs are expected to complete eMedRec, the practical execution of this workflow varies by department and medical service. For example, some surgical services have a full complement of NPs who manage patient care based on the physician grouping during business hours. This means that they conduct all admission, transfer and discharge eMedRec for patients who transition through any of those phases of care during business hours. For other medical or surgical services, eMedRec is completed by the resident or fellow on-call. For admissions that occur after business hours, the resident or fellow on call is responsible for completing eMedRec. The temporal nature of eMedRec use as well as other professional distinctions may account for potential variations in participant experiences; however, the focus of this study is eMedRec use which is, for the most part, the same functionality accessed by all participants.

\section{Inclusion Criteria}

Participants included in this study met the following criteria:

- NP or MD including residents and clinical fellows

- Speak English fluently

- Have a minimum of six months' experience using outpatient medication record (OMR) and orders reconciliation manager (ORM) for admissions, discharges and transfers

- Use eMedRec at least four times a month

All participants spoke English fluently because it is the researcher's primary language but it is also the language with which all information is communicated at the study site. A minimum requirement of six months' experience with the technology was set to ensure that participants have overcome the initial learning curve inherent with clinical technology implementation. In previous studies, post-implementation data collection was often conducted at a minimum of six 
months (King et al., 2006; Ward et al., 2011). NPs and MDs have the appropriate permissions to access most of the functionality within the eMedRec system. The requirement to use eMedRec a minimum of four times a month decreases the likelihood that their experiences will be reflective of a learning curve or lack of familiarity with the technology. All participants must have used each component of eMedRec at least once and this includes the OMR to record a patient's home medications as well as the ORM to reconcile admission, discharge and transfer medications. Within this organization, the entire eMedRec process includes the OMR and ORM and the BPMH note completed by the pharmacist or nurse.

\section{Exclusion Criteria}

Participants excluded from this study met the following criteria:

- All nursing trainees

- All medical students

- Staff Nurses including any nurse not in a Nurse Practitioner position Specified trainees and staff nurses have been excluded because they do not have the required permissions to access the key features of the software that will be examined within this study. Also, the lived experience of this study will include the admission, discharge and transfer processes via eMedRec and trainees and staff nurses do not experience this entire process in their work.

\section{Data Collection}

I conducted semi-structured, conversational interviews with the participants. A semistructured interview format allows the researcher to generate open-ended questions or topics in advance to be covered during the interview (Roulston, 2008). Employing a conversational style of interviewing allowed me to elicit deep, meaningful data to address the research question while 
affording the participants the opportunity to influence the direction of the conversation (Roulston, 2008; Van Manen, 1990). In fact, Van Manen (2014) discusses the importance of ensuring that the questions are aimed at ascertaining the lived experience of the individual by asking pointed questions about their experience with the phenomenon. To ensure that my questions were consistent with the aim of this research study, I posed what Van Manen (2014) describes as specific, detailed questions to each participant that guided the direction of responses while maintaining the conversational flow of the interview. See Appendix E for interview guide.

Van Manen (1990) suggests that the conversational interview in hermeneutic phenomenology is not merely the collection of participant anecdotes and experiences but a collaborative between the participant and researcher to interpret and assign meaning to the phenomenon. To achieve this, I began each interview with a few casual questions to allow the conversation to flow naturally. I also began the interview by sharing my personal experience with eMedRec. To co-create knowledge with the participants, it was important for me to share how my experiences shaped my perspectives on the phenomenon and have contributed to the pursuit of the proposed research question (Van Manen, 1990).

Prior to recruitment, REB approval from the university and the study site was obtained. Informed consent was completed by each participant. Data was collected via in-depth, semistructured interviews lasting approximately 30-60 minutes each. Two weeks later, a 30 minute follow up interview was conducted with each participant. Informed consent was verbally reaffirmed at the commencement of the second interview. The purpose of the initial interview was to ascertain the lived experience of the participants and to address the primary and secondary research questions. The follow up 30-minute interview is what Van Manen (1990; 2014) describes as the hermeneutic interview to validate my interpretation of the initial interview 
with the participants while collaborating to derive meaning from the experiences that they shared. Van Manen (2014) also suggests that the participants might not be able to interpret their own experiences however, the hermeneutic interview also contributed to data analysis triangulation and thus the rigor of this study.

Field notes were recorded manually during each interview to add context to the transcripts. Reflective journaling was used for the purpose of reflexivity during the research process. Each participant provided written consent allowing the interviews to be audio recorded and afterwards the audio recordings of the interviews were transcribed verbatim. Each interview was conducted in a private conference room at the study site and at the convenience of each participant. As an incentive, I provided participants with a $\$ 10$ coffee card after each interview. Participants were made aware that if they decided to stop the interview while it was in progress or they no longer wanted to participate in this study after showing up for the interview, they would still be provided with the coffee card.

\section{Data Analysis}

Within interpretive phenomenology, the researcher acknowledges and reflects on their beliefs and any pre-existing knowledge relevant to the phenomenon of study (Denzin \& Lincoln, 2011; Van Manen, 2014). In this way, interpretive phenomenology contains the assumption that one cannot completely ignore past knowledge and experiences. As a researcher engaged in phenomenology, I used this knowledge of phenomenology to examine and reflect on my knowledge of eMedRec and its related processes within this organization to allow the voices of the participants to be heard. To capture these reflections, I documented my decisions and processes of thought in a reflective journal throughout the research process. 


\section{Epoche and Phenomenological Reduction}

Van Manen (2014) describes the epoche-reduction as the process of being open to experiencing the phenomenon as lived by the participants and the act of reflecting on those experiences. The epoche-reduction includes heuristic, hermeneutic, experiential and methodological reduction. The heuristic reduction examines the taken for granted assumptions about the experience and centers around the concept of wonder (Van Manen, 2014). For example, technology in modern, western society has become ubiquitous therefore it is easy to make assumptions about its ease of use or usefulness and as such, I approached the data analysis with a sense of wonder and was inquisitive about the mundane and taken for granted aspects of the phenomenon.

Hermeneutic reduction requires the researcher to be self-aware of their assumptions, to isolate their interpretation of the data and to convey this in the phenomenological text to facilitate the reader's understanding of the interpretation of the phenomenon (Van Manen, 2014). In this regard, I reflected on my perspective as an experienced informatics nurse, quality leader and a user of eMedRec at the study setting. During one interview, I reflected on my perception of the workaround described by the participant. I noted in my reflective journal that I perceived that workaround negatively because of my past role as a quality leader; however, because I reflected on that assumption, I could understand the importance of that workaround for the participant. Another assumption that I noted during the hermeneutic reduction was my experience with the eMedRec technology and health IT overall. For example, I did not anticipate that participants would describe as many experiences or assign as much meaning to the training they received as I assumed that any negative experiences would be attributed to the technology 
or other work processes. Reflecting on these assumptions, helped me to better understand the participants' experiences.

The experiential reduction compelled me to examine the experience of eMedRec use at its most concrete level while paying attention to anecdotes and specific examples explored by the participants. For example, when a participant described the steps they used to create or edit a discharge prescription in eMedRec, I paid attention to the concrete steps of the process as they described it and as it appeared in the transcribed text but I was simultaneously aware of the context of the patient story, interprofessional relationships or patient safety lens within which a participant would situate their experience. Using the experiential reduction focuses on the description of the events as presented by the participants versus incorporating lofty abstractions of the phenomenon (Van Manen, 2014, p.225). Lastly, the methodological reduction emphasizes that there may be multiple methods to answer a phenomenological research question; however, the researcher should choose their method based on its relevance to the research question (Van Manen, 2014). While interviews are commonplace in phenomenological research, they are also appropriate for this study because interviews were the most feasible and the 30-60 minute window placed limited time demands on the participants.

Van Manen (2014), describes the epoche-reduction as a preparatory phase leading to the reduction-proper in which the researcher employs the eidetic, ethical, ontological, radical and originary reduction. The eidetic reduction explores what is unique about the phenomenon and aims to describe what the phenomenon is and what it is not while the ethical reduction acknowledges the responsibility of the researcher to convey the participants' experiences accurately (Van Manen, 2014). As such, the study findings include experience-based, rich, 
descriptive accounts of the phenomenon to account for the ways of being for NPs who use eMedRec.

\section{The Four Existentials}

The themes were also be analyzed in reference to the four existentials of temporality, relationality, spaciality and corporeality (Van Manen, 1990; Van Manen, 2014). Van Manen (1990) argues that human beings experience the world in terms of these existentials and the act of questioning, reflecting on and writing about human experience requires an understanding of the phenomenon in terms of each of these categories. The existentials provided a method for understanding the concrete aspects of the data in a conceptual manner as discussed in the experiential reduction. To analyze the data in terms of temporality means that I would need to acknowledge the lived experience of the participants as a selected point in time (Van Manen, $1990 ; 2014)$.

For the first existential of temporality, I would need to acknowledge the lived experience of the participants as a selected point in time (Van Manen, 1990; 2014). For the existential of relationality, Van Manen (2014) encourages phenomenological researchers to understand how individuals are connected to each other in the context of the phenomenon. In the case of this study, the concept of relationality facilitated my understanding of how participants work with other members of the interprofessional team, patients, families and each other, both inter and intra-professionally, as they use the eMedRec system. Van Manen (2014) states that when we analyze an experience based on spaciality, we are asking, "[h]ow do we shape space and how does space shape us?” (p.305). In fact, Van Manen (2014) continues to state that spaciality is not isolated to the physical but can also refer to a virtual space that we cannot perceive with our senses. With regards to the phenomenon of eMedRec use, the existential of spaciality helped me 
to understand how participants navigated the sequence of steps within eMedRec, their virtual space, and how that space reflected their experience in their physical reality of caring for patients.

Corporeality addresses the experience of the body with respect to the phenomenon of study (Van Manen, 2014). For this existential, Van Manen (2014) proposes that human beings often proceed through life with very little attention paid to the way in which the body perceives information. Based on this understanding, I used the concept of corporeality to explore the way in which participants experience eMedRec use with their senses. I originally assumed that participants would describe the esthetics of the eMedRec application but in the absence of that data, I used the existential of corporeality to understand the way in which the NP was required to move between the patient's room and the computer at the nursing station to document in eMedRec. Finally, these existentials were employed to understand the phenomenon at a conceptual level which also helped me relate sentences and other parts of the transcribed text to all of the transcribed interviews. The existentials were not used to categorize the findings, but to facilitate my understanding of them.

\section{Thematic Analysis}

To analyze the data, I employed Van Manen's phases of data analysis and referred to Braun and Clarke (2006) after each phase of analysis was complete. For this eMedRec study, the Braun and Clarke approach to thematic analysis complemented the phenomenological reduction described by Van Manen (2014) as it is practical, yet not prescriptive and it has been employed in other interpretive phenomenology studies such as Smith and Osborn (2007). The Braun and Clarke phases included: 1) becoming familiar with the data; 2) generating initial codes; 3) searching for themes; 4) reviewing themes; 5) defining and naming themes; and 6) producing the 
report (Braun and Clarke, 2006). A form of data analysis triangulation was achieved via input from my thesis committee and the secondary interviews with participants. My thesis committee members and participants had the opportunity to validate my interpretation of the data and provide their own interpretation when necessary. I transcribed the data independently which began the process of analysis for each interview because it allowed for immersion of the data. As I listened to the audio recordings while transcribing the text, I reflected on what the participant meant as they described their experiences and documented detailed notes to facilitate my understanding. Once I finished transcribing an interview, I listened to the interview again to double-check the transcripts for accuracy and to compare the transcribed text to my field notes. I listened to the audio recordings of the interviews on several occasions during data analysis and even during the writing of the findings to ensure that I accurately conveyed the description and the meaning of the participants' experiences.

Van Manen (1990) describes thematic analysis as “...the process of recovering the theme or themes that are embodied or dramatized in the evolving meanings and imagery of the work" (p.78). A selective or highlighting and holistic approach was used to analyze the transcribed text. The holistic approach entails reading and analyzing the transcribed text in its entirety; focusing on all words and sentences to ensure that no detail of the interviews is missed (Van Manen, 2014). Furthermore, I enacted a selective approach, whereby I read and re-read the text to determine if there are specific sentences or portions of sentences that stand out with the goal of understanding what these phrases reveal about the phenomenon (Van Manen, 1990; Van Manen, 2014).

After this level of analysis, I composed what Van Manen (1990) refers to as "linguistic transformations" in which notes about the text are constructed to facilitate its interpretation 
(p.96). These linguistic transformations in the form of short sentences and paragraphs facilitated my phenomenological understanding of the text. Once I identified themes from the interview transcripts, I met with the participants a second time for what Van Manen (2014) refers to as the hermeneutic interview in which the participant and I discussed their first interview and together derived meaning from their experiences. Participants did not describe new experiences in any of the hermeneutic interviews; in fact, many repeated stories they told in the first interview and confirmed or clarified my proposed interpretation of their experience.

I occasionally used probing questions asking the participants to explain why they felt the way that they did or how they arrived at an understanding of their experience. In this way, the participants and I engaged in the co-construction of meaning associated with their experience of using eMedRec at the study site (Van Manen, 2014). It is important to note that although the purpose of the hermeneutic interview is to reflect on the transcribed text, as the researcher, Van Manen, cautions that participants might not be adequately prepared to interpret their experiences. Furthermore, I provided space for the participants to challenge my interpretations, to offer their own interpretations and to feel free to acknowledge if they they could provide an interpretation of their experience.

\section{Cognitive Mapping}

The final tool of analysis is cognitive mapping. This method of analysis was used to facilitate my understanding of the interconnected nature of the themes and to provide a visual representation of the participants' description and interpretation of their experience. Northcott (1996) noted that "[c]ognitive mapping generates a picture of the constructs and ideas of individuals and of groups...[and]...combines the researcher's views with those of the respondents" 
(p.458). This understanding of cognitive mapping is congruent with interpretive phenomenology and facilitated the articulation of the co-constructed meaning of the experience.

For this interpretive phenomenology, I analyzed the data using a comprehensive, layered approach including the epoche, a structured, thematic analysis and cognitive mapping. These analysis strategies were employed in this study to arrive at a final interpretation of the data in congruence with the philosophical foundations of this research,

\section{Establishing Rigour}

Rigour within qualitative research is a contentious topic due to the post-positivist lens through which the term is viewed. Lincoln (1995) suggests traditional tools of rigour such as validity, reliability and generalizability aim to eliminate bias in the pursuit of an objective truth; which from an interpretive, qualitative perspective cannot be attained. Lincoln (1995) continues this discourse contending that even the qualitative categories of transferability, credibility, dependability and confirmability are inappropriate measures of quality due to their alignment with equivalent quantitative categories that are philosophically incongruent with qualitative inquiry.

In fact, many scholars suggest abandoning these rigid categories aimed at defining the quality of interpretive or naturalistic research because qualitative inquiry is better understood as occurring on a continuum versus a dichotomous entity to quantitative research (Denzin \& Lincoln, 2011; Lincoln, 1995; Sandelowski, 1993; Van Manen, 2014). In other words, applying criteria to qualitative research that parallels that of quantitative can be problematic because of the varied paradigms under which any given qualitative study may be situated. Specifically, Sandelowski (1993), is concerned with the notion of dependability as a parallel to reliability because if reality is socially constructed and therefore multiple in its versions, then no two 
researchers will interpret data from the same respondents in the same way. Also, the need to replicate a qualitative study is not relevant because of the temporal nature of human phenomena under investigation (Sandelowski, 1993). For example, when I ask participants about their lived experience with eMedRec, I am asking them at a specific time and place in their experience. These participants may provide very different responses if asked the same questions by the same researcher one month or even one day later because human experiences are inherently temporal and context-bound (Van Manen, 1990).

Due to the challenges of conventional qualitative categories of rigour, I selected the de Witt and Ploeg (2006) framework to guide the quality of this study. After extensive literature synthesis, de Witt and Ploeg (2006) created a framework for evaluating the quality of interpretive phenomenological nursing research. The proposed framework incorporates aspects of Van Manen's work, categorized as “expressions” that resonate with the philosophical and methodological stance of this study. These expressions of rigour are balanced integration, openness, concreteness, resonance and actualization (de Witt and Ploeg, 2006).

\section{Balanced Integration}

Balanced integration is the first of five expressions of rigour as defined by de Witt and Ploeg (2006) and includes three main characteristics. Firstly, the researcher must clearly articulate the philosophical underpinnings of the study as well as how this philosophy is suitable for the research topic and the researcher (de Witt and Ploeg, 2006). I have identified interpretive phenomenology based on the work of Van Manen (2014) as the methodology for this study as well as its guiding philosophy. I have also provided an overview of my position as a researcher, eMedRec user and other roles within the organization of study to demonstrate how the philosophy of the study is an appropriate approach for me. 
Secondly, the study's philosophical concepts, methods and findings must be discussed in-depth and seamlessly within the text so that the methods and findings are not discussed in absence of their philosophical contexts (de Witt and Ploeg, 2006). While writing the text of this study, I endeavoured to consistently link the interpretive nature of this study to the interview, transcription and analysis phases of this study. Lastly, the text must display a "...balance between the voices of the study participants and the philosophical explanation" (de Witt and Ploeg, 2006, p.215). I embedded the philosophy of this study within the written text by directly citing it and by offering an interpretation of the NP experience that reveals their way of being via the care of their patients and families.

\section{Openness}

The second expression of rigour is Openness. de Witt and Ploeg (2006) describe openness as an explicit and systematic method of articulating the process of making decisions throughout the course of the study. Openness in this context is not be confused with the sense of wonder that Van Manen refers to in approaching the epoche-reduction during the data analysis phase. In the context of qualitative rigour, openness addresses the researcher's ability to provide an adequate audit trail and clear articulations of research decisions so that the reader has a clear understanding of the how the research findings were arrived at. de Witt and Ploeg (2006) noted this potential confusion between openness as a way of reporting versus interpreting the data in the limitations of their article; however, I employed openness by using tentative, non-prescriptive language to convey the findings and implications of this research.

\section{Concreteness}

Concreteness is the third expression of rigour and it states that the researcher must present the study findings as relevant to the reader as well as within the context of the 
phenomenon (de Witt and Ploeg, 2006). In other words, the reader needs to be able to simultaneously appreciate how the study findings and the phenomenon of study fit in relation to their practice. Many scholars agree that there is a need for research to be relevant to the real world and it is particularly important in a practice-based profession such as nursing (Sandelowski, 1993). To achieve this I used rich, descriptive writing to convey the experiences of the participants. In addition, I provided examples of eMedRec use via direct quotes and scenarios detailed by the participants. I strived to bring the study findings into the context of the reader's life world by providing context to the examples (Van Manen, 1990; Van Manen, 2014).

\section{Resonance}

The forth expression of rigour is resonance. Derived mainly from the work of Van Manen (1990), resonance refers to the effect that reading the study has on the reader whereby the reader arrives at an epiphany about the findings (de Witt and Ploeg, 2006). In their analysis, de Witt and Ploeg (2006) provide a quote in which a participant compared the inception of Alzheimer's to that of an appendectomy. In this way, I have provided a rich account of the transcribed interviews via direct quotes and I used my reflective journals to account for the moments during data analysis when I began to understand key aspects of the phenomenon. By reporting those moments when I experienced an epiphany about the data, I aim to have the reader experience a similar enlightenment about the essence of the phenomenon of study.

\section{Actualization}

The final of the five expressions of rigour articulated by de Witt and Ploeg (2006), actualization, is challenging to evaluate. Actualization is described by the notion that the interpretation of the findings does not end at the reporting of the study (de Witt and Ploeg, 2006; Sandelowski, 1986). To address this, I have written the findings of my study in a way that 
makes it open and amenable to future interpretation. I have attempted to achieve this style of writing by not making finite declarations about the data and the phenomenon in general. I will consistently use reflexive language and refer the reader to my positionality as a factor in the interpretation of the data.

\section{Reflexivity}

It has been suggested that reflexivity is the defining characteristic of qualitative research, in which researchers reflect on their impact on the research process and make explicit their role in the co-creation of knowledge (Finlay, 2002). Throughout this text, I have employed a reflexive writing style to allow my research decisions and methods to be open for scrutiny. I want to ensure that I clearly articulate my positionality as a Registered Nurse at the study organization and as such I have experienced the phenomenon from varying perspectives. As mentioned earlier in the chapter, there are many aspects of technology use that I may take for granted as a technology user and informatics professional. In addition to technology, there are aspects of the NP scope of practice that I may take for granted because of my close collaboration with NPs in both a clinical and informatics context in the past. I documented these reflections to better understand my interpretation of the findings of this study. The proposal of the topic and question for this study is based on my varied experiences and therefore my position as a researcher, clinician and informatics nurse are clearly articulated in this text to allow the reader to fully appreciate the context within which this study was developed, analyzed and reported.

Being reflexive allows the research consumer to better understand how this study may or may not fit within their practice environment and as such, potentially empowers the reader to make decisions about the quality of this work (Van Manen, 2014). In fact, Lincoln (1995) argues that no research study can claim to ascertain the entire truth of a phenomenon as it is always a 
partial truth conveyed based on the positionality of the researcher and the context of their work. As a result, I used reflective journaling as a strategy for maintaining an audit trail of my research decisions as well as a reflexive account of the entire research process.

\section{Ethical Considerations}

Anonymity cannot be achieved in this study therefore the following paragraph details the strategies for ensuring confidentiality. Throughout the study, no demographic information was stored on the audio recording files and participants were assigned a number to ensure confidentiality. At no time were the audio recordings or transcribed data stored in the same location as the legend. The legend was stored on an encrypted USB key in an envelope in a locked drawer at the site PI's research office. The interview data was stored in an encrypted file on the student researcher's laptop located in a locked home office. Numbers were assigned to participants and no patients were directly involved in the study. None of the members of the research team had access any patient-specific, personal health information (PHI) for the purposes of this research.

Signed consent forms and participant contact information for study findings were stored in a locked filing cabinet in the PI's research office. Data from the audio files as well as transcribed data will be kept for seven years in accordance with study site policies. After this period of time, audio files will be destroyed by deleting all copies of the files from the USB key, home computer/laptop including the trash bin in the computer. All paper files will be disposed of in the hospital's confidential bin and then destroyed as per hospital process for confidential documents. Files shared with the thesis co-supervisors did not contain the names of any participants and were distributed via email using the university email addresses. Data files used by the student researcher were transported via an encrypted USB key. The student researcher had 
primary access to the data while the thesis co-supervisors and site PI had access to the deidentified data for the purposes of providing guidance throughout the research process.

The methodology employed in this research study was described including its theoretical foundations, sampling strategies as well as data collection and analysis processes. Researcher reflexivity as well as methods for establishing rigour were also discussed. The use of interpretive phenomenology as a foundation provides a humanistic lens through which to view the findings of this study. The following chapter includes the results of this study and exemplars of the phenomenon. 


\section{Chapter 4 - Findings}

\section{Introduction}

The purpose of this interpretive phenomenological study was to gain insight into NPs' lived experience with eMedRec usage and its related meaning. In this chapter I present the findings of this study including five major themes that emerged from the data: 1) Caring for the patient and family, 2) Enacting patient safety, 3) Practicing within the professional role and scope, 4) Wading through the system and working through the process, and 5) Learning and unlearning overtime. Themes and subthemes are discussed with the support of quotes from the participants. In some cases, the quotes have been grammatically modified from the original verbatim transcription to facilitate ease of reading and understanding. This chapter concludes with a summary of the findings.

Figure 1 illustrates the major themes articulated within this chapter including the relationships that exist between and among each theme. The sub-themes are illustrated separately in Figures 2 to 6. Major theme one: Caring for the Patient and Family, for example, is represented by the largest circle, encapsulating the other circles, due to its central presence in the NP eMedRec experience. Caring for the Patient and Family emerged as a foundational experience of eMedRec use, as participants attached the greatest meaning to the care they provided to their patients. Diagrammatically, the size of the circle, symbolizes the depth and meaning associated with the experience of eMedRec as reported by the participants. Therefore, Enacting Patient Safety is smaller than Caring for the Patient and Family, but larger than the remaining themes because while the participants attributed great richness and depth to the meaning of patient safety, caring for their patients remained fundamental to their eMedRec 
experience. In this context, depth refers to the full scope of the experience as described by the participants and richness refers to the amount of detail they provided.

The remaining three themes are comparable in size due to their similarity to each other in terms of the richness of the experience and the related meaning ascribed to each by the participants. Finally, the overlapping circles within the larger circle represent the interconnectedness between themes in relation to the shared experience of using eMedRec for NPs based on my analysis of the individual experiences. Figure 1 also illustrates how eMedRec connects the themes to each other via the central theme of Caring for the Patient and Family because eMedRec's main purpose is to support patient care. It is important to note that Figure 1 is meant to depict the shared NP experience at a particular moment in time and I acknowledge that the lived experience is fluid and fluctuates over time.

Figure 1 The Lived Experience of NP eMedRec

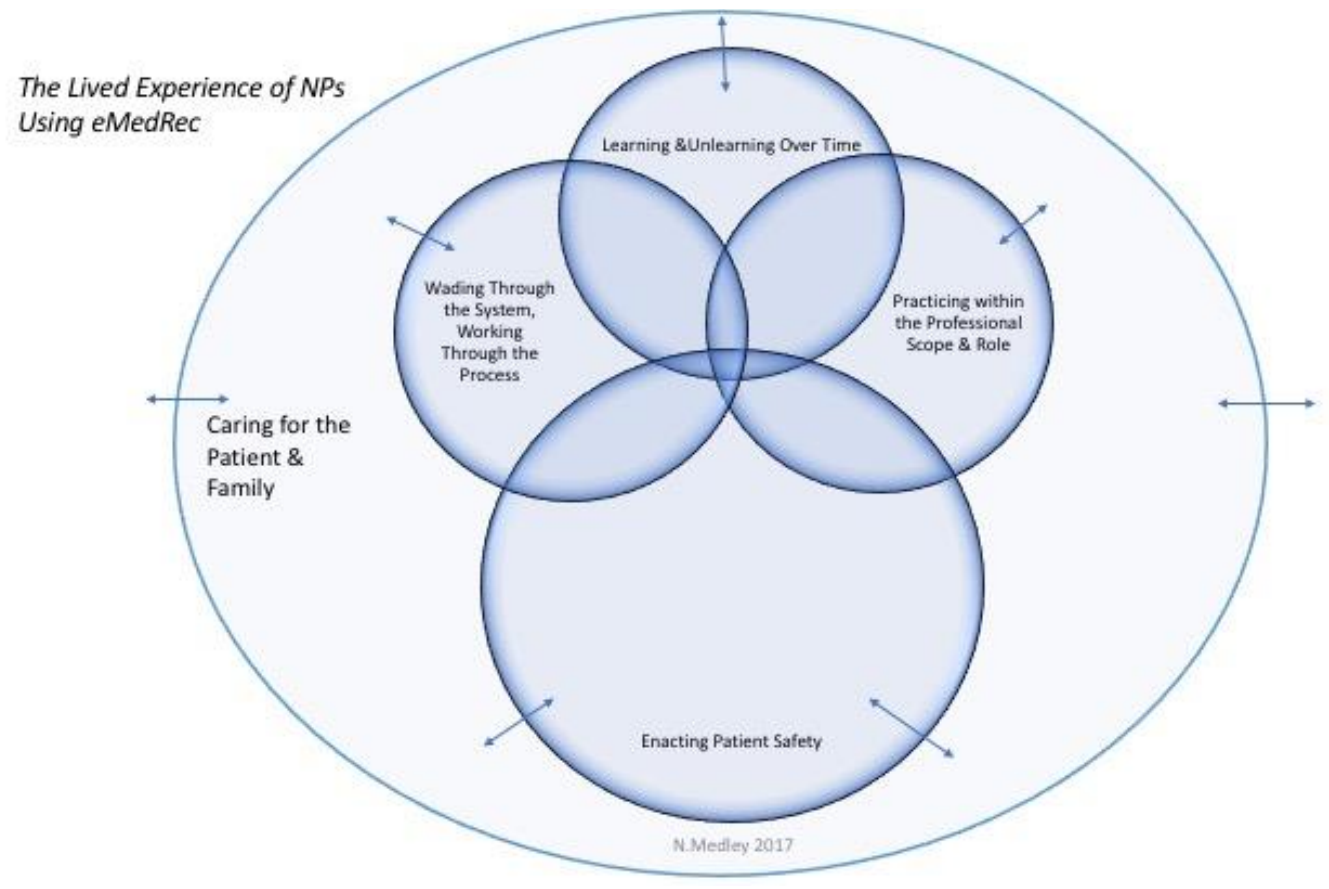




\section{Theme one: Caring for the Patient and Family}

This theme of Caring for the Patient and Family is an articulation of the centrality of direct patient care in the professional lives of the participants because, whether describing workarounds, their learning process or interactions with their colleagues, participants situated their experiences with eMedRec in relation to the care of the patient and family. As a pediatric hospital, the study site enacts family-centred care as its philosophical foundation and includes the care of the patient and their family as one unit which was exemplified in participant accounts of how they provided care for patients and their families. The following sub-themes illuminate the interpretation of Caring for the patient and family: 1) adapting to the complexity of patient care, 2) communicating with the patient/family or healthcare professionals (HCPs), and 3) being accountable for patient care (refer to Figure 2). Supporting quotes will be used to articulate the significance of and the interrelationships between the themes and sub-themes.

Figure 2 Caring for the Patient and Family

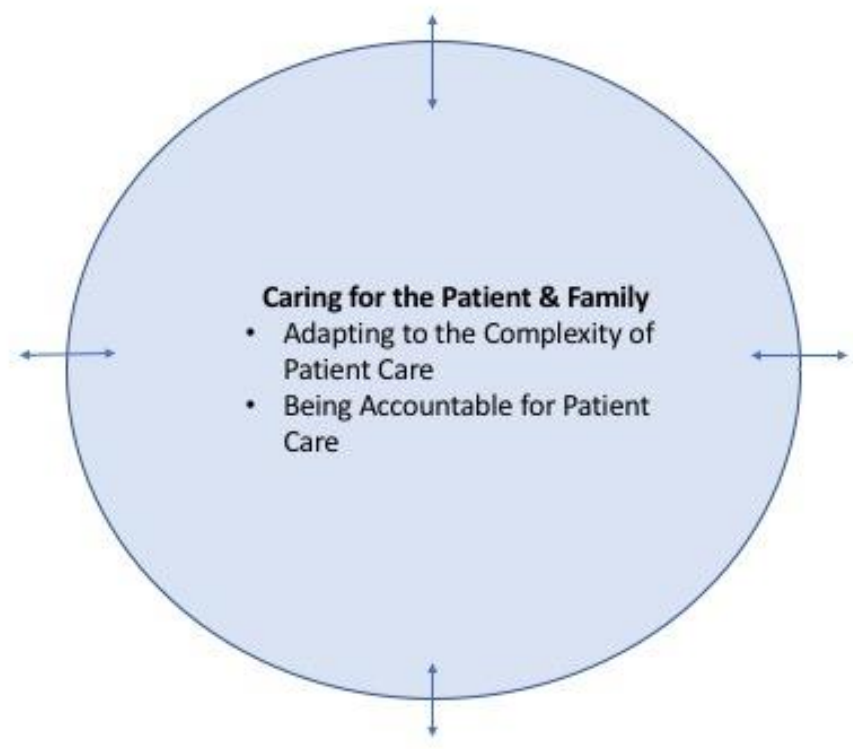




\section{Sub-theme: Adapting to the Complexity of Patient Care}

In a tertiary level acute care environment, NPs may be responsible for managing a variety of complex physiological and psychosocial patient-care concerns. Throughout the interviews, it became evident that the complexity associated with eMedRec use was impacted by the process of caring for the patient and their family. In response to whether eMedRec was a complex process, one participant stated "Simple and straight forward patients, no. It's just part of the thing but if it's a complex kid, it is a lot...you have to be focused for sure." - NP1(Nurse Practitioner \#1). Complexity of the care process can be due to the number of medications the patient is taking, their various diagnoses including co-morbidities, their related care needs and atypical medication regimes.

Participants conveyed that the number of medications taken by the patient shapes the complexity of the eMedRec experience which is exemplified by the following quote: "Ya...the really complex kids with tons of meds can be really tricky..." - NP1. Conversely, two or three medications are perceived to contribute to a more straight-forward eMedRec experience for the NP. "So...usually...it's pretty easy...it's pretty straightforward, so most of the time babies...they're always started on antibiotics so they always come in on Amp and Gent or Amp and Tobra and we continue that [referring to the admission eMedRec process]." - NP6. In this scenario, NP6 is conveying that when patients are admitted with only a few medications that are continued on admission, there are fewer steps to complete within eMedRec. Another NP stated, “...but generally, there's not a lot of meds but the other patients...that I work with are patients that are very complex and they come from ICU [intensive care unit] and so there's always a transfer medrec and that gets tricky..." -NP1. 
This statement from NP1 identifies that complex patients who are transferred from the ICU also make for a complex transfer eMedRec experience. In the follow-up interview NP1 stated that the transfer medrec workflow was more confusing for her because of how the medications are displayed in the transfer medrec module compared to admission and discharge.

The dynamic nature of the admission, discharge and transfer processes was also noted by the participants as a factor contributing to the complexity of eMedRec use.

"But often that plan for discharge changes and...the discharge reconciliation has already been performed so you have to go back the very next day when the actual discharge will eventually happen and re-reconcile and if the prescription has already been entered and not printed, it's really challenging to actually go back and re-order it and print it." - NP3

Another NP adds to this understanding by explaining the unpredictable nature of the discharge process and its impact on the eMedRec experience. This experience is particularly complex when the patient is being discharged to another facility and not the patient's home.

"I have had patients who the discharge reconciliation was already completed in preparation for the baby to be discharged and then you know, things happen. The referral hospital calls back and no longer has a bed. Or...something happens and the medrec's already done." - NP6

The examples provided by NP6 and NP3 are indicative of a dynamic patient care process that involves unexpected changes. These changes in the discharge process, for example, lead to multiple changes in eMedRec to ensure appropriate medication management for the patient. The key challenge experienced by NP6, NP3 and other NPs in this study is that making changes to 
existing data in eMedRec is extremely difficult because of the various steps required; therefore, variations in the patient's plan of care are not easily addressed in eMedRec.

Other patient care complexities encountered by NPs include the varied medical needs of the patient and family. One example is the need to adjust a patient's medications over the course of an admission and how this action may impact the discharge or transfer reconciliation process in eMedRec.

"So [the patients] may start out with Morphine PCA [patient controlled analgesia] and then over the next three days, while they're in the hospital they've been changed to...oral Morphine. And then oral Morphine is not working so they switch to oxycodone let's say..."-NP1.

These changes in the patient's medication plan over the course of an admission and transitioning into the discharge phase impact discharge medrec as NP1 describes that residents often “...wanna be proactive and prepare [the prescription] ahead [of the discharge]...”. NP1 described the changes in inpatient pain management as an example of the non-linear nature of the patient care workflow. The eMedRec workflow, however, seems to follow a more linear path as NPs describe a system that permits minimal deviation and contributes to a more challenging experience for the NP.

Complexities within the family unit may also contribute to the NP's ability to obtain the best possible medication history required in the admission phase of eMedRec.

“...[I]t was challenging cause...you're questioning if there was some [psychological diagnosis] with the parents and ...mom was giving some of her own meds....and we didn't have some of the meds that mom was supplying so there was a huge risk of error..." - NP2. 
In the previous excerpt, the NP's process of using eMedRec as a tool to facilitate care for the patient was challenged, in that the mother's health limited the NP's ability to ascertain an accurate medication history. It also added an additional layer of complexity during the admission process for this patient. Specific to eMedRec, NP2's example also identifies the need for a system that is reflective of the non-linear nature of pediatric care within a tertiary care hospital. NP's do not simply ask questions about the medications and receive a response suited for entry into the eMedRec fields. In fact, NP2 also mentioned that it could take a range of minutes to days to accurately complete eMedRec due, in part, to the family context as well as pre-existing patient-related data such as comorbidities. These challenges may contribute to a more complicated admission process resulting in incomplete information in eMedRec; leading to poor communication of critical patient information to other HCPs within the patient's circle of care.

Others note the challenges of preparing unique protocols for patients with specific diagnoses.

“...TT]here are many of our medicines that we give, for example Septra for PCP [pneumocystis pneumonia] prophylaxis, are only given 3 days a week. So looking back and figuring out 'is this child a Monday, Tuesday, Wednesday or a Friday, Saturday, Sunday patient." - NP4.

In this excerpt, the patient requires the medication three days per week which is not a common medication schedule. NP4 continued to express that the process for creating this type of prescription in eMedRec is very difficult because, when the hard copy version of the prescription is printed from the system, the atypical medication frequency is difficult to understand for patients and families as well as other HCPs such as pharmacists. This creates challenges to 
patient safety for those dispensing the medication. It also impacts the care of the patient at home as the family resumes the role of primary caregiver upon discharge and are provided with a copy of the discharge summary containing the discharge medications.

In summary, Adapting to the Complexity of Patient Care is a sub-theme of Caring for the Patient and Family because the medical and psychosocial realities of the patient-family dyad contribute to the NP experience of using a eMedRec and are at odds with the linear nature of the technology's workflow. This sub-theme also helps to illustrate how the NP is required to adapt to these various patient-care realities while eMedRec is not always as adaptable.

\section{Sub-theme: Communicating with the Patient, Family and HCPs}

The admission, discharge and transfer process is variable, non-linear and requires skilled communication on the part of the NP and other HCPs to ensure safe and effective medication management. Within the patient's EHR, eMedRec is configured in modules for the admission, discharge and transfer medrec processes. The medication information contained within each module informs decisions made by the prescriber, but is also reviewed by other HCPs; therefore, incomplete or inaccurate eMedRec information may lead to erroneous medication orders affecting the patient's plan of care. In addition to the HCPs, the patient and the family are also part of the patient's care team. As such, they are asked to provide medication information upon the patient's admission to the hospital. The patient and family will also be entrusted with the appropriate post-discharge care at home by referring to medication information on the discharge summary. For these reasons, participants have described eMedRec as a communication tool for themselves, other HCPs and the patient and family. There are times when communication via eMedRec may not be effective which will be discussed later. 
The NP and interprofessional team are required to communicate effectively with each other via clinical documentation within eMedRec. One NP describes the complexity of transitioning a patient to home and the adequate communication required to ensure the patient's medications are prescribed and administered appropriately upon discharge.

“...quite often [when] the patient's been prescribed for asthma some inhalation therapies, so Ventolin and Flovent puffers and...the dosing is slightly different than what you would like them to go home on...so their criteria for discharge is when they're tolerating their medication every 4 hours but their doses are often a little bit higher [as an inpatient] so you would need to change that in the medication [for discharge reconciliation]. And often the previous provider has maybe completed the reconciliation but not done that....so you need to go back in and add additional clinical information obviously." NP3

NP3 provided this statement as an example of how important it is to ensure accurate information is entered into eMedRec as it is also a communication tool between the inpatient medical team, the patient and family and the outpatient pharmacy via the prescription and discharge summary. In fact, NP3 continued by describing the discharge summary as “...most helpful because it's a communication that the parents receive into their hands...Cause often you give them a prescription, they give it to the pharmacy and they don't know what they're on...". The accuracy and completeness of the essential information in eMedRec is important as a communication tool for all members of the team and the patient/family upon discharge.

While there are noteworthy challenges to communication experienced by NPs who use eMedRec, NP2 discussed the information communicated via eMedRec as a benefit for obtaining a thorough medication history. 
“...it's also good 'cause [eMedRec] shows me ... 'why was this patient on Morphine in the past?' So it helps my history-taking...to see a bit of the history there because sometimes families leave out details or on EPC [electronic patient chart/old history] or [the current EHR] ...there's information missing..." - NP2

In this scenario, NP2 describes the information contained within eMedRec as a prompt for further questions during the admission health history. Thus, NP2 as an experienced clinician employs their critical-thinking skills to use the historical information contained within eMedRec to facilitate further communication with the patient and family. The information is exchanged between eMedRec, the NP and the patient/family which is ultimately beneficial for the patient. This previous example also reflects how intertwined the subthemes of communication, patient complexity and accountability are within the overarching theme of caring for the patient and family.

\section{Sub-theme: Being Accountable for Patient Care}

The NP has an advanced level of clinical and critical-thinking skills and the result is an increased sense of accountability for the care they provide to patients and their families. Accountability in the context of Caring for the Patient and Family is how NPs directly or indirectly acknowledge the impact their work has on the health and well-being of their patients. The participants expressed an acute awareness of how their actions related to eMedRec during the course of the patient's care can result in the NP's accountability for quality of care for that patient.

An example of how NPs demonstrate accountability for the care they provide is when they ask questions beyond the explicit fields that need to be completed in eMedRec. They use the tools such as eMedRec as a foundation for critically evaluating the patient's health needs. 
“...[W]hen you ask the questions 'what medication is your child on?' ... it shouldn't end there. It should say what medications is your child on, did they take anything else, did they take anything over the counter? - NP1. In this quote from NP1, the NP articulates the challenge of relying solely on eMedRec fields for the medication history as it is lacking in follow-up or probing questions that illicit greater depth of information. By asking additional questions beyond what is asked in eMedRec, NPs take responsibility for ensuring a thorough medication history is obtained.

NP6 also discussed her focus on using eMedRec to protect the patient from errors of duplication. In this scenario, NP6 is referring to medications that are known to have significant side effects, such as ototoxicity, when given in large doses.

“...[I]f you've got for example a cephalosporin like gentamycin or tobramycin, you don’t wanna be giving a double dose of that. So, you really have to know when that medication was last given. And that's important and I think when you have the [medication] reconciliation...it forces you to look and make sure that you know when the last medication [was] given." - NP6

NP6 typically admits babies to the hospital from an external referring facility and as such, these patients have been receiving care in another hospital. Because of the facility-level transfer of care, NPs and RNs need to be vigilant about ensuring they know the last time these medications were given at the referring hospital and thus, they are accountable because the consequences of not being vigilant can be severe.

While it is important to review eMedRec to obtain key information about the patient, this cannot be completed in the absence of a face to face assessment which participants identified as their priority. This continues to demonstrate their accountability for safe and effective patient 
care. One NP discussed the importance of a face to face assessment with the patient versus clinical documentation in eMedRec. "So sometimes the admission process could take a while. But...the paper work is...for me and most people, are always done last and the baby's cared for. And so it's not a delay in care for the baby." - NP6. This statement is significant for understanding the NP lived experience with using eMedRec because it is an example of how NPs prioritize eMedRec within the context of their entire work process. NP6 believes their direct, hands on assessment and care of the patient is the primary task to be completed and all documentation second. While this is understandable, the documentation in eMedRec translates to orders that are required by nursing and other interprofessional team members. This presents a paradox, because while NPs prioritize direct patient care, that care can be delayed or deferred without up to date medication documentation which ultimately risks patient safety. In this way, being accountable for patient care is interconnected with the theme of patient safety.

\section{Theme two: Enacting Patient Safety}

Patient safety was discussed in some capacity by every participant and its foundational place within the context of patient care was evident in each of the interviews. Patient safety is a central topic of concern at this organization due to a hospital-wide effort to significantly reduce preventable patient harm by 2018. The importance of patient safety is also interconnected with all other themes because medrec in general and eMedRec specifically was implemented with the purpose of improving patient safety via accurate medication management at points of transitions in care. The sub-themes of double-checks, correcting errors, workarounds and accountability are reflective of the interface between the eMedRec technology and the work of the NP in the context of patient admissions, discharges and transfers. 


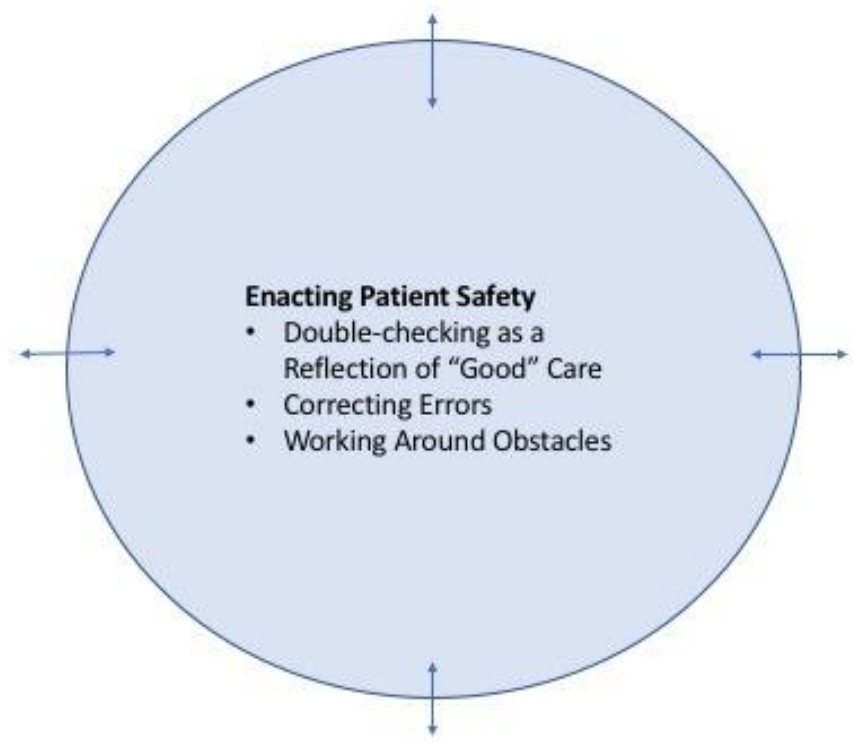

\section{Sub-theme: Double-checking as a Reflection of "Good" Care}

During the interviews the participants first articulated their need to double-check information within eMedRec when describing the admission process. One participant discussed the process of double-checking as a necessity when relying on eMedRec for patient care information. “...[I]t's still not a given that that's accurate... Because [the patient] may have been to another institution so [we] always have to double check... dosing and frequency just to make sure it's accurate. Cause that's how errors happen" - NP3. This statement is significant because it provides insight into the NP's lack of trust in the system and the data contained within it. While it is professionally responsible for NPs to critically examine information entered in any computer system for patient care, this quote from NP3 seems to highlight an awareness of how vulnerable eMedRec is to human error. In this case, the double-checking is an extra step in the 
process which duplicates a process that can be addressed by EHR software with good clinical decision support functionality.

One NP identified that they usually complete eMedRec while they are on the inpatient unit but “... usually I'll double check everything when I'm in my office”- NP2. Doublechecking appears to be an essential step in the eMedRec process for NPs in this organization. Furthermore, one NP articulates the double-checking as, not only an aspect of eMedRec use, but a unique phenomenon reflective of the NP role in an acute care environment. "And I think as a Nurse Practitioner, it's a different role, we're not a physician and so we tend to double check, triple check, you know... and really, making sure that those orders especially medications are perfect..." - NP6. This notion of perfection related to medication management in the pursuit of patient safety may be one reason why NPs feel the need to double-check information entered in eMedRec.

\section{Sub-theme: Correcting Errors}

Participants shared their experiences regarding correcting errors made within eMedRec. They conveyed that this need was in the interest of patient safety and ensuring the communication of the most accurate information about the patient. The errors discussed were either made inadvertently by the participants or their colleagues but each participant, once made aware of the error, felt personally responsible to ensure that the error was corrected. The following is an example of an error inadvertently entered by a NP or MD.

“...[I]f the fellow or myself have entered [a patient-supplied medication to be taken in hospital] and forgot to click 'patient's own med', we actually have to go back to the actual order, right click...modify order... unorder or whatever it is....and then sort of reorder it and click the box... so there's additional steps just to modify that order when 
we're not changing the dose, we're not changing the med, it's just telling pharmacy [not to dispense the medication]..." - NP2

This example highlights how challenging it is to correct errors in eMedRec due to the number of steps the NP must go through. At times the process is cumbersome and they resort to calling the pharmacist instead of correcting the error themselves in eMedRec.

When asked by the interviewer, one participant confirmed that there is a sense of obligation to correct errors in eMedRec. “...I think I feel accountable in the fact that if I see it [the error], I'm gonna correct it" - NP1. In most cases, the participants discussed the challenges associated with correcting errors and these corrections were often required at the point of discharge and specifically within the discharge medrec or prescription writer modules of the software. One participant described an incident when they had difficulty correcting errors made in the system.

“...[O]ne of the residents, when they ordered a medication, they had put a comment with the medication and when I was discharging the patient I wanted to take the comment off because it wasn't applicable anymore and I could not take the comment off." - NP6. This experience as described by NP6 provides an example of how challenging it is for NPs to make corrections within the eMedRec system. It also relates, however, to the previouslymentioned theme of Caring for the Patient because the NP's concern in this scenario was about providing the patient/family with accurate information upon discharge. The example provided by NP6 is an illustration of how interconnected the themes of Caring for the Patient and the Enacting Patient Safety are as well as how the sub-themes of accountability and communication are interwoven. 


\section{Sub-theme: Working Around Obstacles}

One of the obstacles within eMedRec that was consistently identified by participants is the discharge reconciliation process including the use of the prescription writer module. NPs within Ontario are not permitted to prescribe controlled substances due to regulations stipulated by the College of Nurses of Ontario (College of Nurse of Ontario [CNO], 2016). Within eMedRec, however, NPs have the same level of access to the prescription-writer as MDs, yet because of CNO regulations, they cannot follow the workflow as intended by the software. This fact was discussed consistently throughout the interviews. The following excerpt from NP4's interview is an illustration of how this professional practice limitation results in a workaround. “...[U]sually what I will do is go ahead and enter [the order for a controlled substance] into the medrec, which is technically not what I'm supposed to be doing, but I think it's important that it be there from a safety standpoint....so my name is attached to the discharge summary but my name isn't attached to the prescription for that....I won't enter the controlled substance until I've printed off the prescriptions for everything else and then go back and then make the hand-written prescription for the controlled substance." NP4.

This workaround described by NP4 allows the NP to ensure that the controlled substances appear on the discharge summary but not on the electronic prescription with the NP's signature. The key insight in this workaround is the barrier imposed by scope of practice regulation that is not reflected in the eMedRec workflow and the NP's desire to communicate the discharge medications appropriately with the patient and family via the discharge summary.

It is also important to note that within this interview, NP4 explicitly articulated that she is aware that this workaround is not the current standard of practice but there is a professional 
conflict between doing what is correct and successfully completing the discharge prescription in a timely manner. Adding to the understanding of this conflict experienced by NPs, another participant described a workflow by which they bypassed the eMedRec process entirely.

"And sometimes I do all the orders first and then I reconcile after I'm done and I think the process is that I'm to do the medrec right away which makes perfect sense. So...so what I just said is just a user issue more than the way it's set up." - NP6.

In this example NP6 acknowledges that their workflow is a decision not reflective of the acceptable standard in this case. By ordering the medications first and completing the reconciliation after, NP6 negates the patient safety features of the reconciliation process but this workaround was selected because it posed less of a challenge to complete. In NP6's case, it was much easier to enter the order within the CPOE module as a starting point compared to the cumbersome process encountered the admission medrec module. It is possible that these orders are entered this way to maximize efficiency for the NP. This workaround could indicate that there needs to be a more efficient admission medrec workflow to ensure that the NP does not spend large amounts of time trying to navigate the system.

\section{Theme three: Practicing within the Professional Scope and Role}

Throughout each interview, the participants described their work within eMedRec but also detailed the interconnectedness of other HCPs with whom they work. For some participants, their successful use of eMedRec was highly dependent upon the pharmacist and others shared the eMedRec workload with MDs or had clearly delineated roles that differed from those of the MD. Coordinating care and collaborating with other HCPs represents the interprofessional nature of eMedRec use as well as the different ways in which NPs work that are based on the roles of each $\mathrm{HCP}$ on the team. Being accountable for professional scope and role 
highlights the organizational and regulatory policies that affect the NP's experience of using eMedRec. Both sub-themes, as seen in Figure 4, combine to shape the way in which NP's practice their professional role and scope within eMedRec.

Figure 4 Practicing with the Professional Role and Scope

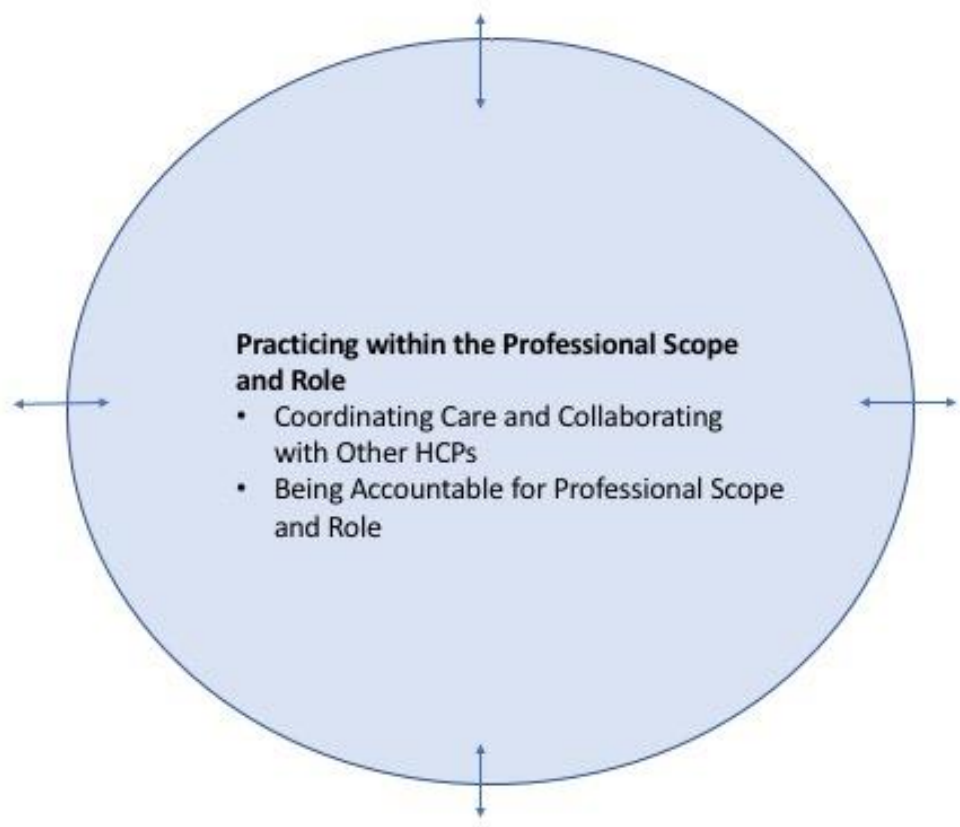

\section{Sub-theme: Coordinating Care and Collaborating with Other HCPs}

In one department, interprofessional collaboration for the purposes of eMedRec is typically between the NP and a fellow. Consistent with the experiences of other NPs in this study, each HCP in NP4's department is responsible for the care their own patients. In the following example, NP4 conveyed the way in which the role of the fellow and the NP differ for this department. "So we created a model... a system that the NPs would be more involved with the patient so the fellows could do more reading of the [diagnostic tests]..." - NP4. In response to my probing questions, NP4 confirmed that this role allocation results in the NP's greater involvement in the eMedRec process. It may be that NPs are educated to employ a holistic, case 
management-like model of care, but it is also possible that although fellows are board certified MDs, they are assuming a learning role within this organization. As such, fellows require practice to refine skills and competencies that overlap with those of the NP, such as reading diagnostic tests.

In addition to their coordination of care with MDs, NPs discussed the importance of the pharmacist as a key collaborator in the eMedRec process. Many participants cited how much they rely on the department's pharmacist to ensure eMedRec is completed accurately. The following quote is an example of how essential the pharmacist is in the process. "And when [the patient] ha[s] I think it's six or more meds...our pharmacist will always do their medrec review...so that's wonderful!...because we go through that together because some of these meds I'm not as familiar with..." - NP1. This example of the pharmacist and NP collaborating during an admission illustrates how patient-related complexity is mitigated via interprofessional collaboration. It also links the sub-theme of collaboration to its major theme of practicing within professional role and scope which connects to Caring for the Patient and Family. These examples of coordinating with the MD and collaborating with the pharmacist help to convey the nuanced relationships between HCPs and their professional roles that ultimately impact the NP's experience of using eMedRec.

\section{Sub-theme: Being Accountable for Professional Scope and Role}

Scope of practice has a significant impact on the level of accountability placed on NPs. As discussed within earlier themes, the roles they hold within their organization based on hospital culture and policies also impact the NP's experience of being professionally accountable. The following quote exemplifies how the NP's accountability changes based on differences in scope of practice between HCPs. 
"So the residents will take their share. They can't order chemotherapy so they will ask one of us to. Or the fellow or a clinical associate or one of the Nurse Practitioners if somebody needs to go home with an order for oral chemotherapy." - NP4

The residents in this scenario are unable to order chemotherapy medications because they do not have the appropriate certification. This barrier for residents is encountered within the CPOE module but NP4 is specifically referring to the discharge reconciliation portion of eMedRec. This means that within eMedRec, residents would be able to reconcile all medications except for chemotherapy and would need to ask for assistance from another provider as such as NP4. The eMedRec system does not restrict the resident from ordering chemotherapy or NPs from ordering controlled substances as these restrictions are practice-driven. Thus, NPs need to request assistance from MDs when prescribing controlled substances and anticipate that they may need to assist residents with their prescribing as well. This process requires NPs to have a keen awareness of their scope of practice limitations as well as those of other prescribers as they use eMedRec.

Scope of practice restrictions that are not accounted for by eMedRec pose a challenge for NPs, however, one participant referred to the different roles within eMedRec as beneficial for establishing appropriate accountability. "So I think that there's multiple levels of responsibility which eMedRec has been able to instill. So, on all levels - so everybody's accountable which before I think with the paper system was less than effective." -NP3. In this section of the interview with NP3, she conveyed that the implementation of an electronic process also introduced a more clear role delineation because of the specific aspects of the software that are restricted to prescribers by security types. In this way, a greater level of responsibility is placed on the prescriber, NPs and MDs, than in the past because they now must complete the discharge 
medrec which was not previously required on paper. Also, NPs and MDs share the responsibility for entering the patient's home medications into the OMR in eMedRec whereas the nurse was primarily responsible for this in the paper system.

Although there are specific security settings for nurses, pharmacists and NPs/MDs, there are other aspects related to the NP experience of being accountable that need to be considered. Determining whether the NP or MD is responsible for completing eMedRec is based on their level of involvement with the patient/family, scope of practice and time of day as NPs typically do not work on call hours and residents and fellows do. Throughout the interviews it was apparent that eMedRec is not always completed in full by the admitting provider because it is possible to complete an admission history and order inpatient medications without actually entering home medications in the OMR and reconciling them with the inpatient orders. In the following quote, this NP describes their frustration when eMedRec has not been completed for their patient.

“...[A]t night there [are] two people on and they're generally of course the fellow and another physician and they get lots of admissions at night. Even if they only had one [admission]...my experiences [have] always been it's never the night medrec that is done. And then of course it's frustrating cause then I come on days and I'm having to do the medrec for somebody else." - NP5

While the experience of having to obtain the best possible medication history hours after the patient was admitted can be frustrating, NP5 also expressed a statement conveying her understanding of why this takes place. “And I get why they don't do it, because there's two of them on - the place is crazy ...I'm telling you medrec is gonna be near the bottom of my priority list." - NP5. So, while there are different roles and responsibilities related to completing 
eMedRec, other factors such as high patient care needs and limited resources impact how HCPs, including NPs, use the system.

\section{Theme four: Wading Through the System and Working Through the Process}

Workflow is articulated in this study as Wading Through the System and Working

Through the Process to represent the pattern of NP work behaviour as influenced by the features within eMedRec and established organizational culture and policies (refer to Figure 5). The term "wading" was used by a participant to describe her need to explore large volumes of information in the patient's chart upon an admission, discharge or transfer. The term connotes a labour intensive process whereby the NP sifts through the entire chart at a level of depth sufficient to obtain information to move forward with the next step in caring for the patient. Similarly, wading through the system encompasses a process by which NPs dedicate much mental and emotional effort as well as their time. The related sub-themes of coping with technological complexity and normalizing workarounds help to convey the challenges that NPs encounter within eMedRec as well as how they feel about those experiences. 
Figure 5 Wading Through the System and Working Through the Process

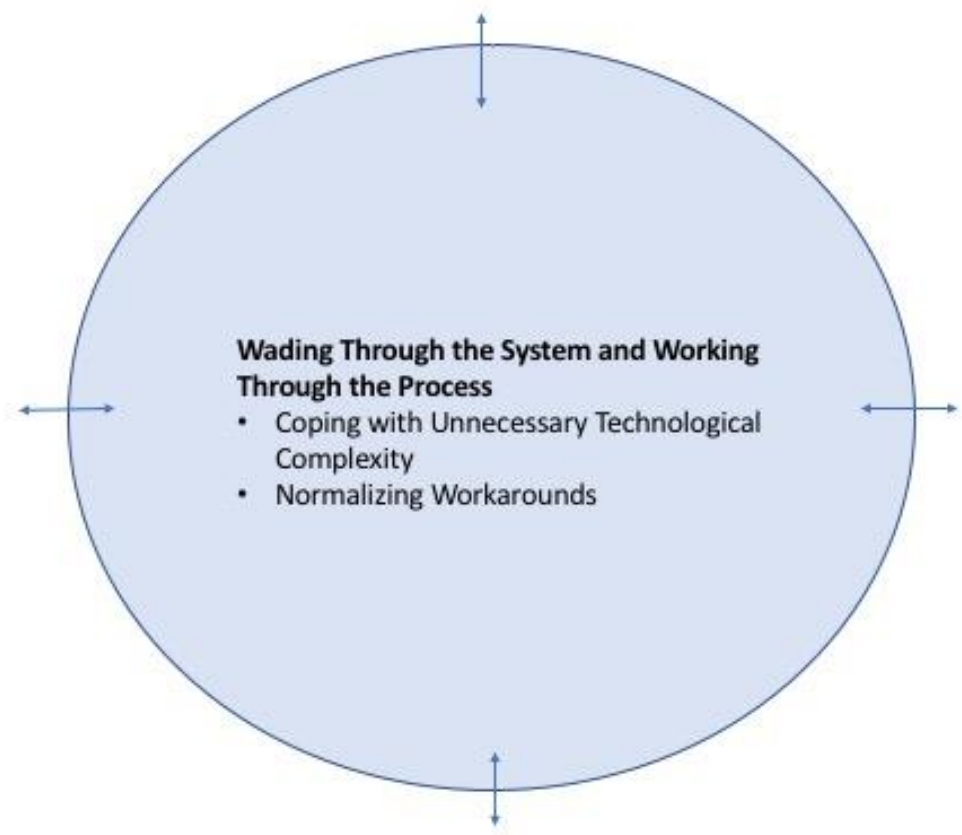

\section{Sub-theme: Coping with Unnecessary Technological Complexity}

As a major sub-theme, technological complexity is specific to wading through the system because it potentially shapes the way the NP thinks through the admission, discharge and transfer processes but it also influences the NP's decision to employ a workaround. Throughout the interviews participants described eMedRec as “... a complicated process when it doesn't need to be..." - NP5. Similarly, other participants have said “...there's...so many...intricacies and you need to be quite detail-oriented for it". - NP2. Interestingly, in the follow-up interview NP2 confirmed the meaning of this statement. She indicated that there is a large amount of mental effort required to complete eMedRec but also stated that providers should devote sufficient attention to the eMedRec process because of its critical nature. This understanding of eMedRec and mental effort can be articulated as the notion of balance. Based on suggestions from participants, this balance within the eMedRec process can be achieved with a system that 
provides enough fields and prompts to complete thorough documentation for the patient but not so many as to cause fatigue and frustration for the NP.

When there are too many details for the NP to process within eMedRec, it can lead to frustrations. One of the intricacies mentioned by the participants is the difference in language used to convey the frequency with which a medication should be given. This difference is evident in the way home medications are entered in the OMR compared to the actual reconciliation/CPOE module in which the inpatient orders are created.

“...[S] o under the outpatient medrec $[\mathrm{OMR}]$ when you enter in their medications and you put the timing, the choices you have are numerous, but instead of BID, TID or every day it's 'two times a day'...it's not the same format as it is when you go to order it." - NP2. This need to translate the medication frequency between two different sections of the same software is an unwanted step in the NP workflow and adds to the complexity of the entire admission process. This also contributes to confusion and frustration for NPs trying to complete their critical work in an efficient and timely manner. There is also the possibility that errors could occur if there are different formats for entering information within the same system.

In addition to differences in the format of medication frequency, medications often bear a different name within eMedRec as evidenced by the following quote.

“And...the other thing I find frustrating is that...if I'm discharging a baby on vitamin D for example, and of course it comes up with the official term for vitamin D...it doesn't say vitamin D. But you click it and there's thirty choices...I'm like 'good heavens!'. Now I know just to go to the bottom one and it will transfer over but when you're first learning and you see thirty choices and you're like 'what on earth?!'... 'what am I supposed to...like...come on!"” - NP5 
In this example, vitamin $\mathrm{D}$ is listed as cholecalciferol in the discharge medication list and there are a variety of concentrations of the medication to choose from. Not only do NPs need to ensure that cholecalciferol is the correct name for vitamin D, but they also need to ensure they select the concentration of the medication from a long list of options. Based on the previous quote from NP5, this is a frustrating part of her discharge workflow within the system as it is not straightforward. In fact, NP5 described eMedRec as not intuitive and when I probed about this in the follow-up interview, she conveyed that comfort with the system was gained by repetition and she defined comfort as being able to navigate the system in a logical flow and timely manner. Further, NP5 learned to memorize the location of the option she needed to select in the drop-down list instead of being able to effectively navigate the system; therefore, she adapted to the system instead of the system adapting to NP's practice.

Similarly, another NP mentioned how challenging it is to have more options than they feel is necessary during the discharge eMedRec process.

“...[B]ut I don't need all those choices. In fact, that is I think increasing...a risk of error too to have a million choices when you really are like... 'no ... why would I change anything?' I just want what was already there.” - NP6

In this example, NP6 is referring to the discharge process in which they are reconciling the patient's existing inpatient medications with the medications prescribed for discharge. The frustration illustrated here is that the discharge reconciliation provides several medication concentration options for the NP to create a discharge prescription. NP6 feels however that changing the concentration of a medication upon discharge is not part of her normal workflow; therefore, having the option to select many medication concentrations that the patient is not currently taking introduces opportunities for error. The examples provided from the interviews 
with NP5 and NP6 appear to indicate that when presented with more options than necessary, the NP experiences moments of confusion and frustration in addition to concerns about the patient's safety as there is an increased risk of error with excessive options. Also, learning to select a memorized option from a drop-down list as mentioned by NP5, provides insight into how NPs learn to use the technology. The technology's complexity impacts the NP's workflow, patient safety and the process of learning and unlearning which will be discussed later in this chapter.

\section{Sub-theme: Normalizing Workarounds}

The complex nature of eMedRec as cited by the participants can shape the NP's workflow by introducing a different way to navigate through the admission, discharge and transfer. By altering the NP's intended process, the complexity of eMedRec may also lead NPs to employ workarounds to achieve instrumental tasks within their clinical workflow. The following explanation from a participant illustrates how frustration with the intricacies of eMedRec can lead to workarounds.

“...[Y]ou really have to go through different avenues to type in medications that aren't in our system [non-formulary]. So then, it almost puts people in the position of like, 'well they're on these goji berries or whatever medication...let's just not enter it'.... when in fact, these things need to be entered in" - NP2.

In this example, NP2 referred to a challenge encountered when the patient's home medications are not listed in the hospital formulary and therefore, not listed in the CPOE section of eMedRec. Participants discussed this challenge throughout most of the interviews. What NP2 describes is the challenge associated with non-formulary medications and how it potentially leads to workarounds in which medical information may be intentionally omitted from eMedRec. 
Omitting information to avoid a complex series of steps in eMedRec is an example of one type of workaround described in the interviews with participants. Another is the use of an alternate method of documentation including paper-based or electronic. One participant described their use of a workaround to maintain patient information across multiple visits to the hospital.

"So I would then pull the patient's chemotherapy chart...so not their hospital chart but the chart that we track their chemotherapy in...so it's in a binder that's not really part of the medical record but it's how we keep track of where they're at in the protocol...."NP4

In the follow-up interview, NP4 confirmed that this process is a workaround because there is no method of maintaining and retrieving the chemotherapy protocol information within the current EHR. While eMedRec stores medication information, it is not used in the outpatient setting which makes it difficult to effectively communicate changes in the patient's chemotherapy protocol while the patient is an outpatient. Because of this unmet need within the existing EHR and the eMedRec module, a secondary, paper-based chart is used to ensure the continuation of the existing clinical workflow.

Finally, one of the most poignant examples of a workaround related to the complexity of eMedRec workflow occurred when a participant attempted to remove a comment within the discharge medrec. This comment was entered earlier in the patient's admission and was no longer applicable at the time of discharge.

"And what I ended up doing is because the patient needed to be moved [to another facility] and no one could figure it out [how to remove the comment] ... I ended up just like... literally taking a black sharpie and ... [motioning on the table as if to draw a line - 
indicating she crossed out the comment] ...because I couldn't keep the comment in

because the comment would be confusing and it was not appropriate." - NP6

This example illustrates how challenging it can be to correct errors in eMedRec due to the lack of easy to use functionality to facilitate these corrections. It also demonstrates how the NP's commitment to and accountability for patient safety can lead to a workaround. NPs are focused on providing the best care for their patients and completing the work that needs to be done but are consistently faced with obstacles. In this scenario, eMedRec is presented as an unforgiving software component that severely limits the NP's ability to append earlier notations leading her to implement a creative solution that circumvents the existing process. NP6 has many years of experience, spoke of her practice with tremendous pride and conveyed a commitment to patient safety throughout both interviews. The fact that NPs with such high standards and levels of expertise would resort to workarounds they know are not ideal could be indicative of a flaw in the software design or configuration. In fact, NP3 stated that there was a flaw in the process of training NPs in the initial and ongoing use of eMedRec.

\section{Theme five: Learning and Unlearning Over Time}

Across all the interviews, participants described ways in which they learned to use eMedRec and how they endeavour to teach others to use it. While the eMedRec implementation required a significant amount of learning on the part of the participants, the new electronic process also required much unlearning of the former paper-based process. The process of unlearning is based on participant accounts of how they completed medrec in the past and it involves forgetting a former way of doing things for the purpose of learning a new way of being. Learning and unlearning appeared to be an ongoing process throughout the use of eMedRec which shaped the NP's overall experience using the technology. The sub-themes of Adapting to 
the learning curve and meeting the learning needs of NPs (depicted in Figure 6) help to articulate the challenges of learning and unlearning complex technology and how NP learning needs were not met during implementation as well as suggestions for future improvement. Because of the NP's role in facilitating eMedRec training for new clinical learners, overcoming the eMedRec learning curve within the context of learning and unlearning also relates to scope of practice and professional roles of the interprofessional team responsible for completing eMedRec.

Figure 6 Learning and Unlearning Over Time

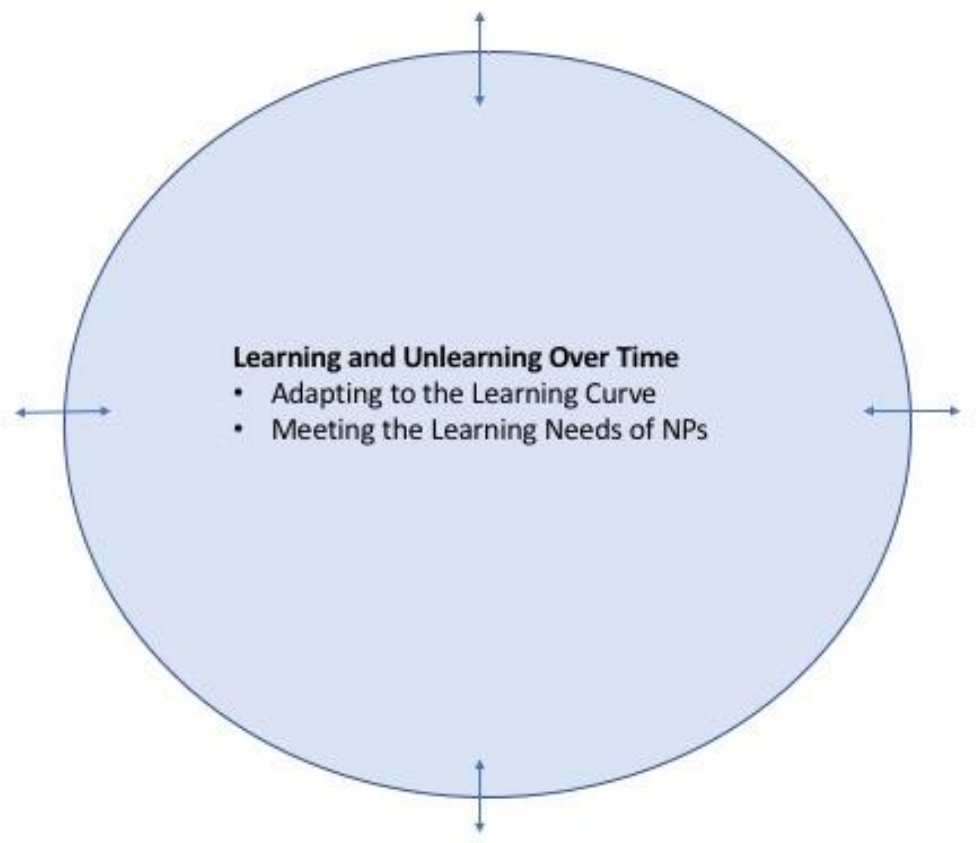

\section{Sub-theme: Adapting to the Learning Curve}

Technological complexity was a significant aspect of the experience of learning and unlearning for NPs as they described how difficult it was for them to learn how to use eMedRec. “...[W]hen I reflect to when we went to electronic medrec, I was so challenged into figuring out how to use it. I didn't feel comfortable using it for at least 6 months." - NP4. Another NP confirmed a similar learning curve during the initial implementation of eMedRec and stated that, 
“...it took me about 3 months to become comfortable with med reconciliation." - NP5. A noteworthy observation from the interviews was that both NP4 and NP5 expressed frustration and disappointment that it took so long to learn how to use eMedRec; however, when I informed them that a three to six-month learning curve is consistent with the literature, they appeared surprised and relieved. It is possible that, due to their high level of expertise, NPs experience extreme discomfort when their role changes from expert to learner which is further complicated by the fact that while learning they are still expected to function in their expert role to deliver patient care.

In addition to discussions regarding their initial frustrations with eMedRec, NP4 and NP5 also understood those frustrations as part of the learning curve. Comparing past and present experience with eMedRec, NP5 explained:

“...[A]nd I did find med reconciliation frustrating at the beginning. The electronic version I found frustrating. I couldn't figure out how to do it at first and...you know, there just seemed to be a lot of options but of course it's clear cut now that you've done it a bit." - NP5.

Similarly, NP4 emphasized the difference between their experience during the learning curve and their current use. "Now it's probably no more time consuming for me than it was with the pre-medrec...pre-electronic medrec. But it took a long time for me to get to that point..." - NP4. It is possible that the participants felt that their learning curve was very steep and took longer to overcome because of the post-implementation support they received. One NP noted that it would have been beneficial to have follow-up education to reinforce some of the initial learning.

The adjustment period during which participants learn to use eMedRec is also a period of unlearning the former paper-based version of medrec. When NPs state that they could not 
understand how to complete eMedRec, even after training, it is concerning because these challenges relate to other themes such as workflow, patient safety and ultimately caring for the patient. In this way, the sub-theme of adapting to the learning curve links the theme of learning/unlearning to all other themes of this study because of how the participants' adaptation to the eMedRec learning curve potentially compromised patient safety.

Many of the NPs shared that facilitating hands-on learning for residents, fellows and other learners, is part of their role as they ensure eMedRec is completed accurately. In addition to being a challenging system to learn, one participant shared that eMedRec is also a challenging system to teach to new staff.

“...And I spend a fair amount of my time mentoring the learners who are coming on [and teaching them] how to use it... and I haven't mastered how to teach somebody how to do it because it's easier to ...it's kinda like driving. You know what you're doing when you have it in your hands but when you're trying to tell somebody what to do, that's a challenge." - NP4

The fact that eMedRec requires additional training beyond the classroom sessions could be due to a variety of factors. Some of the participants cited flawed training and education as one factor, but as NP4 alluded to, it could also indicate that eMedRec's level of technological complexity makes it a difficult application to teach to new users.

\section{Sub-theme: Meeting the Learning Needs of NPs}

An interesting contradiction throughout the interviews is that participants discussed how complex and intricate eMedRec is to use, yet also state that it is much easier now that their learning curve has plateaued. One NP attributed their challenges with eMedRec use to the initial education they received. 
"So...really trying to work through the system on your own or asking colleagues who are not always available unfortunately... Learning from other people's mistakes... is not the best way to learn and still to this day, there still are components of that system that are not as easy to use..." - NP3

In this scenario, NP3 felt that much of their learning took place once they started to use the technology versus from their in-class sessions. NP3 relied on colleagues or their own ingenuity via trial and error to become proficient. This example also highlights the temporal nature of eMedRec use in that learning and unlearning is present at various stages of the experience; from implementation to on-going use. Finally, this quote from NP3 exemplifies the relationship between technological complexity and the theme of learning and unlearning in the NP eMedRec experience.

Regarding the role of the NP in the learning process, one participant described their previous unit-based orientation that included a brief, formal, decentralized education session for paper-based medrec. "We used to have an orientation with the new residents where we would sit for an hour and everyone would kinda quickly have five or ten minutes to talk about different things and medrec was one of them." - NP1. This approach to orienting new MDs to the department changed to a more centralized approach once eMedRec was implemented and NP1 described this fact as a loss for their team.

This centralized approach to learning eMedRec was critiqued by a participant because of her perception that HCP's require context and opportunities for knowledge application while learning a new technology.

“...[W]hen the program was developed and the health care providers are educated on its use, I think rather than showing them how to use the actual technology I think [they 
should learn] prompts and most ...like always questions that they have to ask...like these "always" questions.... you always have to ask what the medication is, what the dose, what the frequency...I think that piece was lost" - NP3.

In this example, NP3 was concerned about the lack of clinical application provided in the education for eMedRec. One participant acknowledged that she was provided with resources to support her learning after the class but it was not sufficient to meet her needs. "Now they can give you some tools and stuff but there's a zillion pages that they gave you. To be perfectly honest, it wasn't sticking..." - NP5. Based on these two examples, the eMedRec education provided during the implementation did not meet the needs of these NPs because they were unable to apply clinical examples to the use of eMedRec and had difficulty retaining the information they were taught.

For participants from one of the critical care departments, their experience during the eMedRec implementation was different because they were learning how to use an entirely different EHR and were introduced to CPOE for the first time. This learning curve is significant and may have impacted their overall experience of learning eMedRec and unlearning not only the paper-based medrec but also a different EHR. The following statement from NP6 may provide some insights into why some participants experienced poor information retention after the eMedRec education and why their learning needs may have been unmet.

"So my learning need was the notes, how to do a note in [current EHR], how to do the orders because that's the priority. So...so...there was a lot of talk about medrec and I thought...'oh my gosh if I hear any more about medrec... when I need to know how to order... you know vital medications and treatments for the babies..." - NP6. 
In this excerpt, NP6 had specific learning needs that were not prioritized during the training. NP6's statement may also be reflective of how she perceived her professional role in this department and as such prioritized order entry and clinical documentation instead of eMedRec which was an organizational priority at the time. This information is significant because it connects the themes of scope of practice with caring for the patient and learning/unlearning. Based on NP6's scope of practice as enacted in her professional role, she perceived other aspects of caring for the patient as her immediate learning priority; therefore, when eMedRec was prioritized by the implementation team, her learning needs were unmet. This is also a noteworthy example because it highlights the impact that organizational culture or policies may have had on how eMedRec was implemented.

Finally, the theme of learning and unlearning is inextricably linked to the entire eMedRec experience for NPs because of how it shaped their initial impressions of the technology and, consequently, their ongoing use of it. For all HCPs, eMedRec classes were tailored to their scope of practice or professional role because eMedRec workflows within the system were dependent upon the HCP's scope of practice. Out of all the interviews however, one quote gave voice to the emotions felt by NPs as they learned about eMedRec. "It was all one class and ... and it was very stressful" - NP6. In this example, NP6 summarized her feelings about the eMedRec training in the context of her learning priorities. NPs recognize the immense responsibility placed upon them to care for patients and families in a safe manner, using the available technology. Based on previous statements from the participants, one four-hour class did not prepare them to confidently take on this critical responsibility. 


\section{Summary of the Findings}

The lived experience of eMedRec use by NPs within an acute care pediatric context is complex and dynamic. It entails professional roles and responsibilities dictated by scope of practice and workflows influenced by the technology and organizational culture or policies. As clinicians with advanced levels of expertise, NPs express a strong sense of accountability to the patient and family. All participants expressed an appreciation for the importance of eMedRec as a process for ensuring accurate medication management. They prioritize patient safety and use eMedRec, or circumvent it, to ensure effective communication and collaboration with the patient, family and interprofessional team. Contrarily, they also employ workarounds to prioritize patient safety. The use of eMedRec and the employment of workarounds are influenced by patient and technological complexity or a perceived need that is not met by the technology. The overall experience of using eMedRec is influenced by the NPs experience of learning eMedRec and unlearning past processes. This final theme of learning and unlearning plays a significant role because this theme does not only exist in one moment during implementation but dynamically occurs across the entire experience. 


\section{Chapter 5 - Discussion}

\section{Introduction}

Hermeneutic phenomenology, informed by Van Manen (1990; 2014) provided the philosophical and methodological foundations for this research because it allowed for explication of the nuances and taken-for-granted assumptions about technology use in healthcare.

Furthermore, it permitted me to have in-depth discussions with the participants, resulting in rich descriptions and interpretations of the NPs' experiences using eMedRec. In this way, I could engage in the co-construction of meaning with the participants. This collaborative process of assigning meaning to the participants' experiences allowed me to uncover details beyond the explicit, superficial aspects of their work with eMedRec. In this chapter, I begin with a summary of the findings followed by a discussion of the methodological considerations in terms of what is known about this phenomenon and what knowledge has been gained from the results of this research. The three major insights that I gleaned from the themes noted in the findings provide the basis for a summary of the implications for practice, policy and administration and education. I then provide suggestions for future research followed by a conclusion of the lived experience of NPs using eMedRec.

\section{Summary of Findings}

The NP lived experience of using eMedRec at a large pediatric acute care facility is rich with complexity and meaning. This NP experience can be articulated via the major themes of 1) Caring for the patient and family, 2) Enacting patient safety, 3) Practicing within the professional role and scope, 4) Wading through the system and working through the process, and 5) Learning and unlearning overtime. NPs communicate their work with eMedRec via patient stories and attribute much meaning to the patient and family experience as part of their own 
experience. They practice within the boundaries of their scope of practice and role requirements while collaborating and coordinating with their HCP counterparts to ensure eMedRec is complete and accurate with the ultimate objective to ensure that their patients are safe. NPs also navigate the process of patient medication management via eMedRec by sorting through large amounts of varying data within the system and circumventing the standard eMedRec process to ensure fundamental tasks are completed. Finally, the NP experience also includes unlearning old processes such as paper-based documentation and learning new processes such as eMedRec both pre-implementation and on an ongoing basis. I arrived at this understanding of the NP experience via the use of interpretive phenomenology which provided a framework and philosophical lens through which to view this phenomenon. In the following section I summarize the way in which phenomenology facilitated my understanding of the eMedRec use for NPs.

\section{Methodological Considerations}

Qualitative methodology is not commonly used to explore how HCPs use technology; however, some studies using interpretive phenomenology have been conducted within the last five years (Lundvall et al., 2014; Konsuwan and Locsin, 2011). Lundvall et al. (2014) conducted an interpretive phenomenology of radiographers' experiences during the radiography processes of planning an examination, producing the images and evaluating the examination. The authors illustrated the entire radiography process as one in which the radiographer interacts with technology and adjusts to meet the needs of the patient (Lundvall et al., 2014). This radiography study articulates a similar phenomenon to eMedRec use by NPs; however, Lundvall et al. (2014) relied heavily on description as a method of conveying the findings with less emphasis on interpretation and analysis. In contrast to Lundvall et al., my interpretation was enhanced by the 
hermeneutic interview in this eMedRec study and I omitted the practice of bracketing for an authentic application of interpretive phenomenology to explore technology use in healthcare.

This phenomenological approach, informed by Van Manen $(1990 ; 2014)$ gave primacy to the co-creation of knowledge, embracing my positionality as a nurse and my experience, allowing for an authentic understanding of the lived experience. (Van Manen 1990; 2014). This hermeneutic phenomenological approach shed light on context in which the eMedRec experience exists, and allowed for a detailed account of the nuances of this platform and its use. Max Van Manen (2014) stated that "[r]ich descriptions that explore the meaning structures beyond what is immediately experienced gain a dimension of depth" (p.355). These rich descriptions and their associated meanings are some of the fundamental attributes of interpretive phenomenological research (Van Manen, 1990; 2014). Because technology is now ubiquitous in the lives of most individuals in Western culture, our experiences of using technology can be taken for granted. Throughout the interviews with the participants, I engaged in conversations that allowed participants to explore and make sense of the meaning attached to their experiences. In this way, both the researcher and participant gained greater insight into the needs of NPs who use eMedRec. This application of human science methodology allowed me to obtain data that illuminated the way in which NPs enact their professional roles while navigating through a system that compartmentalized their thoughts.

\section{Insights into NP eMedRec Use}

After analyzing the data, I reflected on the meaning of the five themes and how they contributed to an overall understanding of the collective NP lived experience in this context. The following insights that I will discuss are reflective of the meaning associated with the five major themes of this study. There are three main insights gained from studying this phenomenon using 
an interpretive phenomenological lens: 1) The NP Role and eMedRec - the deeper meaning associated with the NP role and how this is enacted while using eMedRec, 2) Understanding the Pediatric Patient and Family - the importance of the patient and family to this phenomenon of study and 3) the Patient Safety Lens - the challenge of implementing technology based on the goal of increasing patient safety. I compare these insights to the extant literature and discuss the implications of each insight within its respective section.

\section{The NP Role and eMedRec}

The first insight is that the NP role is distinctly different from others on the healthcare team although they are often conceptually grouped together with MDs for the purposes of health IT functionality at the study site. While the initial intent of this phenomenology of eMedRec use was to understand both NP and MD experiences, the data that emerged from solely NP participants was beneficial in that I gained an understanding of the unique role of the NP in the context of health IT use. This difference in role and scope was evident in statements from the participants as they discussed their level of responsibility regarding eMedRec. For example, NPs consistently felt the need to double check their work which could be indicative of their lack of trust in the eMedRec system. This lack of trust could stem from errors they have experienced or their difficulties correcting patient data within the system as they described during the interviews; however, it is worth considering that there might be other aspects of the overall NP experience affecting their need to double-check their work in eMedRec. One participant articulated the fact that she is not a physician as a rationale for double-checking which could be reflective of the NP role in the context of an environment in which she may perceive that MD knowledge is valued over that of the NP. 
In a phenomenological study of the NP role, Rashotte and Jensen (2010) noted that NPs at times described that they second-guessed their decisions and felt anxiety about major patientcare decisions. This example of how an NP enacts their role can provide further insight into NP's use of eMedRec due to the parallel of double-checking found within this eMedRec study and that of Rashotte and Jensen (2010). It is possible that the way in which NPs experience their professional roles can ultimately impact the way they use tools such as eMedRec. In fact, Van Manen (2007) explains that the phenomenology of practice is primarily concerned with articulating a connection between "...being and acting...between who we are and how we act" (p.13). Based on this assumption, I propose that NPs who use eMedRec struggle to trust the eMedRec system because, based on their description, there are flawed aspects of the technology that potentially contribute to patient safety risks. At a deeper, more revealing level of analysis however, it is possible that the way in which NPs embody their professional roles and their relationships to and with their MD counterparts greatly influence how they use eMedRec.

In addition to understanding the embodiment and enactment of NP's professional role via eMedRec, examining how NP's use eMedRec via phenomenology allowed me to uncover the misalignment between the NP's way of being and the eMedRec design and configuration. This misalignment could also explain the presence and normalization of workarounds as noted by the participants. Varpio, Schryer and Lingard (2009) noted an interesting exception in their findings that is consistent with the workarounds noted in this eMedRec study: a participant followed up their workaround with conversations with interprofessional colleagues. In the same way, NPs navigate the barriers within eMedRec by enacting a workaround but ensuring that the correct information is communicated to the interprofessional team. Therefore, this demonstrates a 
contradiction in that NPs understand the patient safety implications of a workaround, yet continue to use workarounds while attempting to manage the risk of error.

NPs are often required to adapt to the requirements of eMedRec instead of eMedRec adapting to the workflow of the NP. In reference to technology implementations that focus greatly on the technology versus the work process, Clegg (2000) noted the result of this type of implementation is that "...the task becomes that of designing the social system around the technology" (p. 464). Clegg (2000) continues by arguing that software design should be focused on user workflow. Unfortunately, this method of implementing technology, outside of social systems or with the expectation that social systems will adapt, persists. Based on the data, eMedRec compartmentalizes the patient admission, discharge and transfer aspects of the NP experience which is not in line with the NP's organic way of thinking through these workflows. This misalignment was exemplified by NPs' descriptions of “carrying over" information from one module of the system to another.

In a study discussing the evolution of the Systems Engineering Initiative for Patient Safety (SEIPS), Holden et al, (2013) proposes that task factors such as sequence and complexity are attributes of the task to be completed and these factors influence the overall work system and thus, the processes and outcomes of a healthcare-related scenario. Applied to eMedRec, one task factor that affects the work system is the sequence of the eMedRec workflow. Furthermore, the layout of the admission, discharge and transfer medrec modules impact the sequence of how the NP uses the system. Flanagan et al., (2013) found that issues related to the layout and organization of patient data within an EHR was one of the reasons nurses employed workarounds at one of three sites in the study. Flanagan et al., continued by stating “... providers desired to view patient information differently from the way in which it was presented on the computer to 
better support their work" (p. e63). These findings from Flanagan et al. support the notion that when EHRs, such as eMedRec, are not designed with the user workflow in mind, they may use a workaround to achieve their desired tasks.

Applied to eMedRec, NPs articulating "carrying over" information from one module of eMedRec to another reveals the absence of an intuitive workflow because this technology should follow the organic steps of an admission, discharge or transfer and therefore auto-populate information into other sections of eMedRec as needed. Instead of requiring users to open the Outpatient Medication Record (OMR) to enter the patient's home medications and then enter a completely different module to reconcile those medications with admission orders, eMedRec should flow fluidly from one section to the next while using automation to trigger which sections of eMedRec to enter data in next. The Holden et al. (2013) SEIPS 2.0 model depicts the structures that impact the healthcare process resulting in outcomes related to the patient and the healthcare system. Holden et al. therefore, helps to understand the findings of this study in that NPs interact with eMedRec technology to complete the task of reconciling patients' medications during admissions, discharges and transfers. The factors of patients, families, other HCPs and the technology itself interact with one another and ultimately impact the overall eMedRec process and thus outcomes such as patient safety.

\section{Implications for Practice}

An understanding of the NP role including their professional sense of self and day to day workflow can greatly improve the design and configuration of the technologies they use for patient care delivery. For example, NPs in this study tended to check and double check their work in eMedRec. Thus, it behooves the developer to design applications with a simplified data entry experience allowing for the accurate reporting of patient data that NPs and other HCPs can 
rely on for patient care planning. Clegg (2000) suggests that technology should be designed to effectively contribute to productivity while being reflective of the user and organizational goals. If technologies such as eMedRec are designed with the purpose of providing safe, effective and efficient patient care in mind, then NPs will be better equipped to conduct thorough medication histories and care planning via medication management. It is also possible to decrease NP's need to employ workarounds if technologies such as eMedRec that are designed with an understanding of the NP workflow and role. This understanding of how NPs experience their role is also important for NPs to have as they will be better able to articulate their system design needs to technical or business professionals who are not familiar with their work. For example, NPs consistently referred to the eMedRec system as one that is not intuitive.

\section{Implications for Policy and Administration}

Nurses may hold a variety of positions of leadership in which they are responsible for the development of hospital policies, NP role delineation and the procurement of computer and information technologies to support patient care. It is essential for nursing leaders to understand the NP role including their scope of practice and how it will impact their use of any technology for patient care. With eMedRec, participants were challenged by the cumbersome nature of the discharge workflow and developed workarounds as a result. Those responsible for the procurement of new technologies for a hospital need to ensure that NPs are adequately represented in the decision-making process because, as this study has revealed, NP needs vary from those of other prescribers. If NPs, as people within the work system noted by Holden et al. (2013), contribute to the process outcomes for the hospital via the use of technologies such as eMedRec, then their experiences should to be understood during the decision-making phase of new software procurement. The individual living the experience is best prepared to address the 
needs of that experience and therefore, armed with skills in clinical care, leadership and research NPs can be an asset to an organization embarking on a new health IT investment.

\section{Implications for Education}

Participants expressed disappointment in the training they received during the implementation of eMedRec and some related their current concerns back to their original training. It is important to consider that although NPs, at times, feel a sense of uncertainty in their practice, they are also a group of HCPs who take pride in being comfortable, competent and confident in their practice (Rashotte and Jensen, 2010). It is understandable then, that the transition from competent expert to an uncertain learner in the face of new EHR implementations can make NPs feel very vulnerable and uncomfortable.

Educators and training specialists involved with the implementation of new EHRs need to ensure that the NP role, scope of practice and day to day workflow is accounted for and not just combined with the MD role when developing training. It is important for NPs to be provided with case scenarios relevant to their practice and opportunities to practice prior to implementation to reduce the feelings of stress associated with their shift in expertise during a new EHR implementation. Based on needs articulated by the participants of this eMedRec study, training for technology implementations should include several shorter classes over the course of a few days. Informatics educators can also involve NPs at the curriculum development stage by including a NP representative as they often have knowledge and experience in curriculum development. In fact, Le Grow, Hubley and McAllister (2010) assessed the evolution of the Advanced Practice Nurse (APN) role at a large academic pediatric hospital and noted that APNs, a group that includes NPs, contribute to the advancement of pediatric nursing by

providing education and engaging in curriculum development. NPs may be able to leverage their 
clinical knowledge and graduate-level education with their experience of learning and unlearning eMedRec to contribute to improvements to the way NPs are trained on clinical systems in the future.

\section{Understanding the Pediatric Patient and Family}

The second insight from this study is that eMedRec articulates the experience of the patient and family in a way that effectively reduces their experience to a linear process void of the nuances inherent in a complex acute care environment. While eMedRec is a tool to facilitate the effective management of patient medications and cannot contain every possible scenario that a patient may encounter, it needs to be malleable enough to adapt to the patient's changing condition. In a randomized control trial in which eMedRec was implemented to test for a decrease in preventable adverse drug events (PADEs), Schnipper et al., (2009) found that although PADEs decreased after eMedRec implementation, they were unable to eliminate these types of events. In fact, Schnipper et al., (2009) noted that there were 1.09 PADEs per patient in the intervention group and one of the reasons cited was a “...lack of patient or caregiver knowledge of the preadmission medication regimen..." (p.777). While patients and families should not be blamed for adverse drug events, it is possible that patient and family knowledge can be enhanced by forming collaborative relationships with their healthcare team and hospital administration. By consulting with patients and families and demonstrating how technology is now a major part of the care they receive, healthcare teams can seek valuable feedback from patients and families to influence the design of systems that HCPs use. Patients and their families can also contribute to an understanding of how hospitals can address their medication management needs via technology. This inclusion of the patient and family in conversations surrounding health IT is also in line with Holden et al., (2013) and their understanding of 
complex healthcare systems as they cite the patient as one of the people-related factors in the complex work system of healthcare.

\section{Implications for Practice}

While exploring NPs' experiences of professional practice, Rashotte and Jensen (2010) explicated the NP's desire to maintain direct contact with patients; regardless of their other leadership or administrative pursuits and it parallels the experience of NPs who use eMedRec within the context of Caring for the Patient and Family. Specific to a pediatric setting, Le Grow et al. (2010) explain that the patient and family is the centre of the pediatric NP's practice which aligns well with this eMedRec study findings. The care of patients and their families is the foundation of the NP experience; thereby affecting how they use eMedRec in their practice. The focus on the patient is also evident in the clinical practice of other professionals as noted in Lundvall et al., (2014) as they describe their need to provide extra support for a patient with anxiety for example. The need to understand the patient experience is evident in the NP lived experience with eMedRec. Health informatics professionals and anyone involved with health IT initiatives need to understand and configure NP workflow from a patient perspective if they wish to meet the needs of the NP who will provide care to these patients. Patient portals through which patients could electronically access their medication list, could be employed as a method of engaging patients and their families in the medication reconciliation process while also improving the way in which patients and families manage their medications and communicate with the healthcare team prior to admission.

If the NP articulates the patient as central to their experience of using eMedRec, then it is possible that workflow designed with the patient and family in mind first may be extremely beneficial. For example, for the purposes of pre-implementation requirements-gathering, it may 
be of greater importance to ask an NP, "What does your newly diagnosed patient with leukemia need on their first day of chemotherapy?", versus "What do you do for a patient on their first day of chemotherapy?". This method of gathering health IT requirements is consistent with the data when NPs conveyed a patient diagnosis and/or the circumstances surrounding the workflow they were trying to explain. Van Manen (2007) explains that the phenomenology of practice aims to make sense of the way in which we act in everyday circumstances and helps to form a connection between the way we think and act. Applied to NP's, their experience with eMedRec was intertwined with the experience of the patient and family which was evident in the various accounts of how they cared for the patient and family using the technology.

\section{Implications for Policy and Administration}

Patients are the reason why healthcare technology such as eMedRec exists; therefore, they should be considered in the design, configuration, implementation and on-going use of such technology. Holden et al., (2013) suggests that there should be greater collaboration with patients when trying to understand the sociotechnical implications of technology used in healthcare. Further to that point, I would suggest that patients and families need to be included in the stakeholder consultations when hospital administration considers procuring new technologies such as eMedRec. By understanding patient and family needs, healthcare administrators and other nursing leaders can better understand the system requirements needed to deliver safe and effective care to the patient populations their organizations serve. Also, leaders within the technology sector including experts in human factors science could work closely with patients and families in the development and evaluation of new technology as they are the indirect consumers of the technologies being sold to healthcare organizations. 
Also, health IT developers and HCPs who provide clinical insight into the development of products such as eMedRec, need to consider the needs of patients and families at the design, testing and implementation phases. Specifically, patients and families who choose to incorporate a medication regime that does not adhere to the typical, Western biomedical model are those who may not benefit from the current eMedRec system as participants described how challenging it is to enter medications, including complementary medicines, not listed in the hospital formulary. By providing a wider range of options for entering this data in eMedRec, NPs would be able to easily include complementary medicine in the medication documentation and consider its impact on the patient and their plan of care. Higgins, Larson and Schnall, (2017) conducted a concept analysis to arrive at a definition of the concept of patient engagement to articulate its utility within the nursing discipline. The author's note that the concept of patient engagement has the potential to change organizational practices. To engage patients and families in the procurement and implementation processes, hospital leadership could consult with their patient and family advisory committee and seek feedback from them regarding various health IT solutions that may be implemented. The procurement and implementation of health IT products such as eMedRec are two processes that patients and families can be involved in to inform the way in which health IT is managed.

\section{Implications for Education}

Because NPs assign great meaning to the experience of Caring for the Patient and Family, it is important for this value to be reflected in the training and education they receive during health IT implementations. Stories and case studies related to the patient and family need to be embedded in the training so that the notion of using technology as a tool for patient care is not reduced to a series of mouse-clicks and key strokes. By using case studies within any health 
IT training, institutional policies and standards of practice can be reinforced using current patient care realities. This suggestion should not be limited to the training received immediately prior to go-live, but should also apply to any post-implementation optimization plans as the process of learning and unlearning is temporal and present at various moments in time.

\section{The Patient Safety Lens}

Finally, the third major insight relates to the fact that eMedRec is a system that was implemented with the intent of increasing patient safety but, based on the interviews with participants, eMedRec has introduced new layers of complexity that can potentially decrease patient safety. While there are some features of eMedRec that participants found beneficial, they spoke in greater depth about the risks to patient safety resulting from a cumbersome system that is not adaptable to variable workflows. Campbell et al., (2006) found that new types of errors developed after the implementation of a CPOE module even though the common discourse at the time was that technology can be used as a strategy to decrease the risk of medication errors (Feng, Bobay \&Weiss, 2008; Karsh, Holden, Alper \& Or, 2006). Campbell et al. (2006), also noted that the reason these systems may result in new types of errors is due to a flawed user interface and concerns regarding the configuration of the test and production environments for the technology. I would argue that latter concerns may be more indicative of a flaw in the implementation practices at the study site for Campbell et al. and not necessarily a concern related to CPOE or health IT implementations in general. User interface issues such as the long pick lists and poor data organization mentioned in Campbell et al. are consistent with the findings of this eMedRec study.

The key argument of this insight into eMedRec and patient safety is that theories regarding the patient safety benefits of healthcare technology, in the absence of an understanding 
of the real-life experiences of patients and the HCPs who care for them, can reduce the practice of safe patient care to a robotic experience whereby the person, both the patient and the HCP, become bystanders to the experience. In this way, technology use in healthcare is reduced to binary terms in which the presence of technology translates into patient safety, but based on previous evidence and the findings of this study, this depiction of technology and patient safety does not account for the multiple layers of complexities that may impact a patient at any given time. In fact, Almaberti et al., (2006) noted that deviations from standards of practice occur as individuals adjust to a new process and move closer to the border of safe practice while trying to ensure that they meet productivity and safety expectations. Based on this understanding from Almaberti et al., a new process such as eMedRec may not contribute to increased patient safety without the deliberate management of violations that occur during the evolution of a new process.

Holden et al. (2013) proposes that the revised SEIPS 2.0 model offers researchers, HCPs and health informatics professionals a more robust analytical tool for understanding the impact of the complexities of the healthcare system. When the person, including the patient and HCPs such as NPs, are factored into an understanding of how the entire work system of an acute care pediatric hospital functions, researchers are better prepared to explain why errors persist despite the implementation of technology deemed to be safer. Leveraging the SEIPS 2.0 model with the findings of this eMedRec study related to NP concerns about patient safety, can provide insight into how NP's eMedRec use is affected by their work system, which translates into varied processes including, but not limited to, the selection of workarounds to attain the outcome of safe patient care. In other words, if any part of the work system and processes do not function as expected, patient safety could be compromised (Holden et al., 2013). Therefore, the 
implementation of a health IT solution such as eMedRec cannot, in isolation, address such complexities without considering all factors that contribute to the experience.

The participants in this study demonstrated a keen understanding of patient safety culture and how technology can be of benefit or detriment to patient safety. NPs are experienced professionals and most of the participants described what patient care was like prior to computerized documentation and order entry. It is possible that their level of experience and understanding of the paper-based method of completing all aspects of documentation allowed them to be more discerning about what information to rely on within eMedRec and when to double-check with the patient or family.

In a hermeneutic phenomenology exploring intensive care nurses' experiences managing technology-dependent patients, Alasad (2002) noted that the experienced nurse can balance the technical skills required to manage the technology with their skills for providing patient care. Furthermore, Alasad (2002) conveyed that experienced nurses integrate technology and patient care and are, thus, able to ascertain information from both the patient and the technology to provide safe patient care. I previously discussed the notion that NPs appear to have a lack of trust of eMedRec; however, that can be a useful perception because participants questioned the information they received from the system and employed critical-thinking skills that extended beyond the capabilities of eMedRec. While this may be a normal part of NP practice, their integration of critical-thinking skills in the presence of maladaptive aspects of eMedRec, could explain why they may normalize workarounds in certain instances despite their understanding of patient safety culture. In this scenario, the workarounds may be a demonstration of their criticalthinking skills as they attempt to adjust to the technology. 


\section{Implications for Practice}

Feng, Bobay \&Weiss, (2008) and Karsh, et al., (2006) advocate for health IT solutions that include clinical decision support functionality such as dose-checking and allergy warnings for example; however, those solutions are already included in the current eMedRec/CPOE system at the study site so participant accounts did not reflect a need for these solutions. While the current eMedRec system does contain some clinical decision support functionality, when newer systems are implemented, there is an opportunity to expand on the existing eMedRec functionality by conducting an environmental scan within the hospital to determine the most prevalent type of errors as well as the types of errors that contribute to the greatest harm. If any of these errors can be mitigated or even moderated slightly via clinical decision support functionality, the hospital's health informatics team could prioritize the configuration of such functionality. An example of clinical decision support technology that may be beneficial is a tool within the chemotherapy protocol. Participants could enter the day of the protocol and the system could cross reference pertinent lab values, documented symptoms and other medications the patient is taking to provide the prescriber with suggestions for next steps in the plan of care.

It is important to be aware of the potential for blind, over-reliance on technology to conduct all the critical-thinking of a HCP. Campbell et al., (2006) cautions that over-reliance on clinical decision support functionality may contribute to increased vulnerability for errors if there is an unexpected system downtime. Human beings are an essential aspect of the healthcare system and technology should be used as a tool to facilitate the work of the NP and improve patient care via clinical decision support functions but not in the absence of the critical-thinking skills of expert professionals like the NPs in this eMedRec study. 


\section{Implications for Policy and Administration}

The implementation of an eMedRec system is a significant achievement that moves an organization toward reducing the gaps in medication management that may increase the risk of harm for patients. That said, it is important for policy makers and leaders in healthcare to understand that eMedRec and similar technologies have the potential to contribute to a safer environment for patients and much consideration should be given to the user experience and workflow implications to exploit health IT benefits to patient safety. Clegg (2000) suggests that core principles for designing technology are that the key processes should be integrated and congruent with each other. For example, the prescription writer within eMedRec could be better integrated with the discharge reconciliation module so that NPs and other prescribers do not need to click a separate icon to create a prescription. In this way, an action taken by a user in one aspect of the system could trigger an automatic action in a different yet related section of eMedRec. To further that point, Clegg (2000) also mentions that these technologies and their design should be owned by management and the users of the system. This inclusion of users in the design process may also help to decrease or eliminate what Varpio, Schryer and Lingard (2009) refer to as "forcing the EPR (electronic patient record)" which is a type of workaround that HCPs employed to ensure that the system adapted to their professional workflow p.683.

Specific to this eMedRec study, if users drive the design and configuration of an integrated system, then NPs are more likely to experience workflows within the system that are congruent to the way in which they work. In fact, Tucker (2016) proposed that participants who had greater access to the owner of the process for medication administration, were more likely to employ policy-compliant workarounds instead of workarounds that were a violation of institutional standards of practice. As such, hospital administrators should ensure that the 
implementation of new health IT-related processes should include a process owner with whom users of the new system will be able to confer. For example, organizations could employ health informatics professionals who have a keen understanding of the clinical and IT processes as well as the authority or influence to make changes at the policy-level.

With regards to usability, hospital administrators could work to ensure that procurement policies explicitly state the need for documented usability studies from any health IT vendor from whom they are considering the purchase of new health IT solutions. Administrators should also leverage the skills and expertise of the NP and ensure that they are included in stakeholder discussions regarding the purchasing, configuration and implementation of any new healthcare technology. Including NPs in these conversations from the very beginning will be of great benefit to the individual NPs and the entire organization because if the system is designed with the user and patient in mind then the usability concerns discussed by the participants in this study may be reduced.

\section{Study Limitations}

From a qualitative perspective, this eMedRec study could be enhanced by observational data. Observation of NPs or other HCPs using the actual technology may facilitate a more comprehensive description of the experience for interpretation. Instead, I asked specific questions about the software during the initial interview so that the participants could make clear the processes they referenced. The follow-up hermeneutic interview was very helpful as it allowed me to interpret the data with the participants and clarify scenarios that I was not able to visualize or understand during the initial interview.

While the study participants were all NPs, I also intended to recruit MDs for this study. Having only NPs in this study was beneficial as interpreting the data for one professional group 
allowed me to focus on their unique needs. If this study is conducted in the future with MDs, the researcher should consider that there are many MDs at this study site who are in a learner role which may require different strategies for recruiting them into this study. The NPs in this study received two, ten-dollar coffee cards, but MDs who are in a learner role may respond to recruitment letters more readily with a form of monetary reimbursement. Also, the researcher recruiting MDs for this study should consider that the different types of MDs at this study site include learners and attending MDs who represent various level of hierarchy which may impact their overall description and interpretation of the experience.

It is important to note that the goal of this eMedRec study was to expose the individual and shared lived experience of NPs at the study site and as such, the findings discussed are intended for the context within which the research was situated. While this study was conducted with one professional group, the results may or may not have relevance for other eMedRec stakeholders. For this reason, the recommendations are meant to address the NP experience at the study site from the perspectives of practice, administration, policy and education.

\section{Future Research}

The use of hermeneutic phenomenology informed by Van Manen (1990; 2017) provided a methodology for describing and assigning meaning to the experiences of NPs who use eMedRec. By uncovering underlying assumptions about the NP role, patient care and technology's role in patient safety, there is now a foundation for further exploration into the relationships between various aspects of the experience. For example, this interpretive methodology can be used to explore the MD or pharmacist experience of eMedRec use. Because of the NP's similar prescriber role to the MD and overlapping responsibilities in practice and in eMedRec, it would be beneficial to understand the unique experience of the MD and how 
eMedRec can be improved to meet their needs. The same type of study could be conducted for pharmacists due to their similar use of eMedRec but potentially different overall work process.

The findings of this study could provide the basis for further research exploring whether a relationship exists between user experience and patient experience in healthcare technology. Because of the on-going presence of education in this study's findings via the theme of learning and un-learning, an interventional study could be conducted in which an innovative curriculum is developed based on the recommendations from participants. Pre-implementation training and post-implementation optimization using the new innovative curriculum could contribute to a greater understanding of the strategies to employ when teaching HCPs to use various health IT solutions within an acute care environment.

\section{Conclusion}

In this study, I addressed the research question: what is the lived experience of NPs who use eMedRec and the factors influencing their use of the system. I employed hermeneutic phenomenology to arrive at the themes of: 1) Caring for the patient and family, 2) Enacting patient safety, 3) Practicing within the professional role and scope, 4) Wading through the system and working through the process, and 5) Learning and unlearning overtime. From these themes I analyzed the complex and implicit aspects of the NP role, the pediatric acute care environment and patient safety in medication management. The findings of this study add to the existing knowledge because of a new understanding of how a NP's role as well as the patients and their families contribute to a collective experience of eMedRec use.

The lived experience of using eMedRec for NPs is rich with patient stories of complex diagnoses and interventions that need to be accounted for by the technology to prevent the use of unsafe workarounds. The NP's experience of eMedRec use is temporal and occurs at various 
moments in time, requiring a system that is capable of the fluidity existing in a pediatric acute care environment. The NP's embodiment of the care of patients and their families contributes to their professional sense of self and ultimately how they use tools such as eMedRec to complete their work. NPs interact with eMedRec in a virtual space that constricts their thoughts into compartments not consistent with the way in which patient care is conducted in their practice, yet it provides them with a foundation for ensuring an adequate medication history is obtained. In many ways, their experience is dialectical because while NPs strive to provide excellent patient care as safely as possible, they also employ workarounds when presented with seemingly insurmountable barriers within the eMedRec system. Finally, NPs experience eMedRec through their relationships with their MD and pharmacist colleagues as they negotiate the tasks and plans of care for their shared patients. NP's use of eMedRec is affected by the people they interact with who are involved in with the patient care process, including the patient, family and other HCPs. Their organizational culture and policies as well as features of the technology also affect their use of eMedRec.

To address the needs of NPs and patients and families as discussed in this eMedRec study, health IT professionals could design and configure eMedRec systems with the patient as the priority and in consultation with the NPs, patients and their families. Van Manen (1990) suggests that to conduct hermeneutic phenomenology is to attempt that which is nearly impossible - to convey the way in which human beings experience the world while simultaneously recognizing that any explanation of this experience can never fully capture all its complexities. This phenomenology of the NP's eMedRec use at a large pediatric hospital is my attempt at conveying such complex realities. 


\section{$\begin{array}{ll}\text { Research } \\ \text { Ryerson } & \text { Ethics Board }\end{array}$}

To: Nadine Medley

Nursing

Re: REB 2016-149: Electronic Medication Reconciliation and the Lived Experience of Nurse Practitioners and Physicians

Date: May 17, 2016

Dear Nadine Medley,

The review of your protocol REB File REB 2016-149 is now complete. The project has been approved for a one year period. Please note that before proceeding with your project, compliance with other required University approvals/certifications, institutional requirements, or governmental authorizations may be required.

This approval may be extended after one year upon request. Please be advised that if the project is not renewed, approval will expire and no more research involving humans may take place. If this is a funded project, access to research funds may also be affected.

Please note that REB approval policies require that you adhere strictly to the protocol as last reviewed by the REB and that any modifications must be approved by the Board before they can be implemented. Adverse or unexpected events must be reported to the REB as soon as possible with an indication from the Principal Investigator as to how, in the view of the Principal Investigator, these events affect the continuation of the protocol.

Finally, if research subjects are in the care of a health facility, at a school, or other institution or community organization, it is the responsibility of the Principal Investigator to ensure that the ethical guidelines and approvals of those facilities or institutions are obtained and filed with the REB prior to the initiation of any research.

Please quote your REB file number (REB 2016-149) on future correspondence.

Congratulations and best of luck in conducting your research.

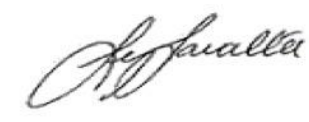

Lynn Lavallée, Ph.D.

Chair, Research Ethics Board

The Following protocol attachments have been reviewed and approved. 
- Interview Guide Final.docx (submitted on: 19 Apr 2016)

- ForClientTranscriptionConfidentialityAgreement[1].docx (submitted on: 19 Apr 2016)

- NMedley Informed Consent.docx (submitted on: 19 Apr 2016)

- NMedley Audio Consent.doc (submitted on: 19 Apr 2016)

- Email Recruitment Letter - NMedley04062016.docx (submitted on: 19 Apr 2016)

- NM Recruitment.docx (submitted on: 19 Apr 2016)

- Recruitment Screening Form NMedley.docx (submitted on: 16 May 2016)

- V2 NM Recruitment 05122016.docx (submitted on: 16 May 2016)

-V2 NMedley Audio Consent 05122016.docx (submitted on: 16 May 2016)

-V3 Email Recruitment - NMedley05122016.docx (submitted on: 16 May 2016)

- Ryerson REB Requirments May2016.docx (submitted on: 16 May 2016)

- V3 NMedley Informed Consent 05122016.docx (submitted on: 16 May 2016)

If any changes are made to the attached document throughout the course of the research, an amendment MUST be submitted to, and subsequently approved by the REB. 
Appendix B: Study Site REB Approval

2016-09-02

Jennifer Stinson Nursing

\section{Research Ethics Board (REB) Study Approval Letter}

REB number: 1000054281 Study Title: Electronic Medication Reconciliation and the Lived Experience of Nurse Practitioners and Physicians

Date of Approval: 2016-09-02 Expiry Date: 2017-09-02

Thank you for the application submitted on 2016-07-28. The above referenced study was reviewed through a delegated process (not by Full Board review). Any concerns arising from this review have been documented and resolved.

The REB voted to approve this study, and your participation as Principal Investigator, as it is found to comply with relevant research ethics guidelines, as well as the Ontario Personal Health Information Protection Act (PHIPA), 2004.

Research Ethics Board hereby issues approval for the above named study. This approval is effective from 2016-09-02 to 2017-09-02. Continuation beyond that date will require further review of REB approval.

The following document(s) have been reviewed and are approved:

1. Protocol: Version 3 - September 2, 2016 [V3 Protocol 09022016 REB Revised Clean.docx (1.0)] 2. Recruitment Poster - June 16, 2016 [NM Recruitment Poster -new06162016.ppt (1.0)] 3. Participant Consent September 2, 2016 [NMedley Informed Consent 09022016 REB Revised Clean.docx (1.0)] 4. Participant Audio Consent [V4 NMedley Audio 
Consent 06062016.docx (1.0)] 5. Recruitment Screening Form 20160620.docx (1.0) 6. Interview Guide - September 2, 2016 [V2 Interview Guide 09022016 REB Revised.docx (1.0)] 7. Screening Form - June 16, 2016 [Recruitment Screening Form 20160620.docx (1.0)] 8. Interview Guide - June 15, 2016 [ V2 Interview Guide 06152016.docx (1.0)] 9. Email Recruitment - September 2, 2016 [Email Recruitment - NMedley 09022016 REB Revised Clean.docx (1.0)]

During the course of this investigation, any significant deviations from the approved protocol and/or unanticipated developments or significant adverse events should immediately be brought to the attention of the REB.

\section{Kathy Boutis}

Dr. E. Stephenson M.D., MSc. REB Chair Arbelle Manicat Emo, RN(EC), MS, NP Paeds REB Vice Chair; Dr. Kathy Boutis, BSc, MSc, MD, FRCPC REB Vice Chair; Rose Gaiteiro, RN, MSN REB Vice Chair

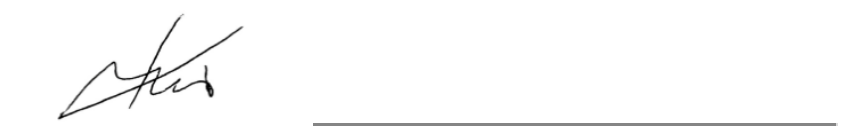

REB \# 1000054281

REB Main Delegated, Page 1 of 2

The REB operates in compliance with the Tri-Council Policy Statement; ICH Guideline for Good Clinical Practice E6(R1); Ontario Personal Health Information Protection Act (2004); Part C Division 5 of the Food and Drug Regulations; Part 4 of the Natural Health Products Regulations and the Medical Devices Regulations of Health Canada. The approval and the views of the REB have been documented in writing. The REB has reviewed and approved the clinical trial protocol and informed consent form for the trial. All investigational drug trials at are conducted by qualified investigators.

Furthermore, members of the Research Ethics Board who are named as Investigators in research studies do not participate in discussions related to, nor vote on such studies when they are presented to the REB. 
Appendix C: Research Consent Form

\section{Ryerson University}

\section{Research Consent Form}

You are being invited to participate in a research study. Please read this entire consent form so that you understand what your participation will involve. Before you consent to participate, please ask any questions to be sure you understand what your participation will involve.

\section{Title of Research Project:}

- The Lived Experience of eMedRec Use Among Physicians and Nurse Practitioners

\section{Investigator(s):}

- Principle Investigator: Dr. Jennifer Stinson RN, PhD, /University of Toronto

- Student Researcher: Nadine Medley, RN, BScN, Master of Nursing Student, Informatics Nurse nmedley@ryerson.ca

- Thesis co-supervisors: Dr. Nancy Purdy RN, PhD, Ryerson University; Dr. Oona St. Amant RN, PhD, Ryerson University

- Thesis committee; Dr. Patrick Neumann, Eur. Eng., PhD

\section{Purpose of the Research:}

This study is being conducted as a partial fulfillment for a Master of Nursing degree at Ryerson University. The purpose of this qualitative study is to provide insight into the processes related to electronic medication reconciliation (eMedRec) at by understanding the lived experience of nurse practitioners and physicians as they use eMedRec. This study aims to address the following research questions: what is the lived experience of eMedRec use by physicians and nurse practitioners at a large pediatric acute care hospital? What are the factors affecting the use of eMedRec by physicians and nurse practitioners?

\section{Description of the Research:}

This study will involve one 60 minute one-on-one interview between you and researcher. The second interview will be 30 minutes in length and will be two to four weeks after the first interview. You will be asked about their experiences using eMedRec via a semi-structured, conversational style interview. All interviews will be recorded with a digital audio recording device and a separate consent form granting the researcher permission to record your interview will be provided. 
- The goal of the first interview is to understand your experiences with eMedRec as well as how you interpret those experiences.

- You will be asked about the following:

- Your role within the hospital and how it relates to eMedRec use

- The process of using eMedRec for patient admissions and discharges

- Your challenges and successes related to your use of eMedRec

- The second interview will be a follow up to the first in which the researcher will discuss her analysis of the first interview with you.

- The goal of the second interview will be to compare the researcher's interpretation of the first interview with your interpretation and to ensure that both parties collaborate to derive meaning from your experiences.

There will be no patients involved in this study, nor will you be required to access patient information in your role as a participant.

\section{Potential Harms:}

We know of no harm that may arise from taking part in this study.

\section{Potential Discomforts or Inconvenience:}

This study will require up to 1.5 hours of your time which we understand may be an inconvenience. Great effort will be made to ensure that the interviews take place within the main hospital campus; however, there may be exceptions due to room availability in which interviews may be conducted in the hospital's research building.

\section{Potential Benefits:}

\section{To individual subjects:}

You will not benefit directly from participating in this study. Potential benefits may be your contribution to research and having your voice heard regarding organizational processes. It is also possible that showcasing the roles and experiences of physicians and nurse practitioners within a large teaching hospital also highlights challenges within these roles. Awareness of these challenges may contribute to improved understanding and inclusion of these professionals during technology implementations within this organization.

The results of the study will be made available to you via knowledge dissemination activities within the hospital. Additionally, the study results will be available to the you upon request.

\section{To society:}

Due to the study's purpose and small sample size, this study is not generalizable to society.

\section{Confidentiality:}

We will respect your privacy. No information about who you are will be given to anyone or be published without your permission, unless required by law. 
Because the researcher is a graduate student, her thesis co-supervisors and the site PI may review your interviews to check on the study; however, all interview data will be de-identified prior to transcription and only the student researcher will know your identity. While thesis co-supervisors and the site PI will have access to de-identified individual interviews, the remainder of the committee will only have access to aggregate data. By signing this consent form, you agree to let these people look at your de-identified interview transcripts.

Published study results will not reveal your identity and a pseudonym will be assigned to your interview data. The pseudonym legend, contact information form and data will all be stored in separate encrypted files.

The data produced from this study will be stored in a secure, locked location. Only the primary investigator will have access to the data and may share the de-identified data with the individuals mentioned above. Following the completion of the research study, the data will be kept for seven years by the principle investigator and then destroyed. Digital files will be deleted and the trash bin emptied and hard copies will be disposed of in hospital confidentiality bins.

We will provide you with a copy of this consent form.

\section{Reimbursement:}

- You will be provided with one $\$ 10$ coffee gift card per interview for their time participating in this study

\section{Participation:}

-It is your choice to take part in this study and you can stop at any time. If any questions make you uncomfortable, you can skip that question. Should you decide to withdraw your participation in this study, all of your data will be destroyed and will not be used in the analysis or reporting of the findings. This study does not have any impact on your current or future relationship with and Ryerson University. Your employment will not be affected whether you decide to participate in this research study or not.

\section{Sponsorship:}

- There is no sponsor for this study and it is being conducted as a partial requirement for a Master of Nursing degree.

\section{Conflict of Interest:}

- Dr. Stinson, and the other research team members have no conflict of interest to declare

\section{Consent :}

By signing this form, I agree that:

1) You have explained this study to me. You have answered all my questions.

2) You have explained the possible harms and benefits (if any) of this study. 
3) I understand that I have the right not to take part in the study and the right to stop at any time. My decision about taking part in the study will not affect my employment at or my relationship with the study investigators or Ryerson University.

4) I am free now, and in the future, to ask questions about the study.

5) I understand that no information about who I am will be given to anyone or be published.

6) I have read and understood pages 1 to 5 of this consent form. I agree, or consent, to take part in this study. I agree, or consent, to take part in this study.

Printed Name of Subject

Subject's signature \& date

Printed Name of person who explained consent

Signature of Person who explained consent \& date

If you have any questions about this study now, please ask.

If you have any questions about this study in the future, please contact:

Nadine Medley, RN, BScN, Master of Nursing Student

Daphne Cockwell School of Nursing, Ryerson University

Email:

Phone:

Or

Dr. Jennifer Stinson, R.N., PhD, Principal Investigator

Email:

Phone:

If you have questions about your rights as a participant in this study, please call the Research Ethics Manager at_ and/or The Ryerson University Research Ethics Board at:

Research Ethics Board

c/o Office of the Vice President, Research and Innovation

Ryerson University

350 Victoria Street

Toronto, ON M5B 2 K3

416-979-5042 
rebchair@ryerson.ca

Please indicate if you would like to be provided with a summary of the research findings. Yes [ ] No [ ]

If yes, please provide your contact information below:

Name:

Address:

Email address:

Telephone:

This information will be kept in an encrypted file, separate from all other data and will be destroyed with all other data within seven years of the study's completion. 


\section{Appendix D: Email Recruitment Letter}

Hello,

I am a Master of Nursing student working under the supervision of Dr. Nancy Purdy and Dr. Oona St. Amant in the Daphne Cockwell School of Nursing at Ryerson University. For my Master's thesis I will be conducting a qualitative study regarding the use of electronic medication reconciliation. Dr. Jennifer Stinson is a member of my thesis committee and is also the onsite principal investigator for this study.

The purpose of this study is to provide insight into the processes related to electronic medication reconciliation (eMedRec) at by understanding the lived experience of nurse practitioners and physicians who use eMedRec. This study aims to address the following research questions: what is the lived experience of eMedRec use by physicians and nurse practitioners at a large pediatric acute care hospital? What are the factors affecting the use of eMedRec by physicians and nurse practitioners?

I am currently seeking nurse practitioners and physicians including residents and fellows to participate in this study. Volunteers must have a minimum of six months' experience using eMedRec for admissions, discharges and transfers at and must use eMedRec at least four times per month. Also, volunteers must not be staff nurses, trainees or medical students.

Participation is voluntary and will involve one 60 minute, one-on-one, audio recorded interview and one 30 minute follow up interview to be held at the hospital. The second interview will be conducted approximately two-four weeks after the first. The initial interview will consist of discussions surrounding your use of eMedRec at for the purposes of admission, discharge and transfer. The second interview will be one in which I will validate the initial interview and subsequent researcher analysis with each participant. You will be provided with a \$10 coffee card for each interview.

Your participation is completely voluntary and if you choose not to participate it will not impact any existing relationship you and I have or your relationship with $\square$ or Ryerson University. Your employment will not be affected whether you decide to participate in this research study or not.

If you are interested in participating in this study, please contact Nadine Medley at

I will respond by sending you an electronic copy of the study informed consent for your consideration, as well as some dates from which you could choose to participate in an interview if that is what you decide. I would be happy to answer any questions you might have prior to confirming an interview time.

Sincerely,

Nadine Medley, R.N., BScN

Master of Nursing Student

Daphne Cockwell School of Nursing

Ryerson University

Dr. Jennifer Stinson, R.N., PhD

Principal Investigator 


\section{Appendix E: Interview Guide}

eMedRec NMedley

\section{Phenomenology Interview Guide}

Introduction

Thank you for participating in this interview. It will be approximately 60 minutes in length and you can stop this at any time. Your participation in this interview will inform my understanding of how Nurse Practitioners and Physicians experience the use of electronic medication reconciliation (eMedRec) at this hospital. Please feel free to provide me with as many details as possible and ask for clarification as needed.

\section{Question \#1}

Can you describe your role as a physician/nurse practitioner with respect to the admission, transfer and discharge processes in the hospital? Describe for me your understanding of your role in eMedRec?

Probing Questions:

- Do you think this impacts your eMedRec use?

- How does this fit with your personal expectations of your professional role?

- Why do you think this is your role?

- Can you explain further?

- Can you provide an example?

\section{Question \#2}

Based on your experience, can you describe the process for using eMedRec? Can you take me through a typical admission, transfer and discharge and how you use eMedRec during each process?

Probing Questions:

- What steps lead you to that section (of the software)? Can you explain in more detail?

- From which department do you typically admit/transfer your patients?

- To which department/location do you typically admit/discharge/transfer your patients?

- Where do you typically use eMedRec? On the inpatient unit? In an office? In the patient's room? Another place in the hospital?

Sentemher? 2016 
- Can you explain further?

- Can you provide an example?

- Why do you think this is the typical process?

- Why do you feel that way?

\section{Question \#3}

Can you provide an example in which you think eMedRec was beneficial for you and/or your patient? In what ways was it beneficial? Are there other benefits?

Question \#4

Can you provide an example in which you think eMedRec was not beneficial for you and/or your patient? In what ways was it not beneficial? Are there other ways in which eMedRec is not beneficial?

Probing Question:

- Are there other factors to consider that contributed to your understanding of the benefits or negative consequences of using eMedRec?

\section{Question \#5}

How would you describe your most challenging experience using eMedRec? How would you describe your most straight forward experience using eMedRec? What do you think contributed to either experience?

Probing Questions:

- Why do you think you found it challenging/straight forward?

- How did this experience make you feel? Why do you think you felt that way?

- Can you elaborate on that point further?

\section{Question \#6}

Are there any other examples of your experiences with eMedRec that you would like to provide? Is there anything else that you would like to tell me that I haven't asked about? 


\section{References}

Alasad, J. (2002). Managing technology in the intensive care unit: The nurses' experience. International Journal of Nursing Studies, 39(4), 407-413. doi:10.1016/S0020-7489(01)00041-4

Amalberti, R., Vincent, C., Auroy, Y., \& de Saint Maurice, G. (2006). Violations and migrations in health care: A framework for understanding and management. Quality and Safety in Health Care, 15(suppl 1), i66-i71.

Asaro, P., \& Boxerman, S. (2008). Effects of computerized provider order entry and nursing documentation on workflow. Academic Emergency Medicine , 15, 908-915.

Bates, D., \& Gawande, A. (2003). Patient safety: Improving safety with information technology. The New England Journal of Medicine, 348(25), 2526-2534.

Braun, V., \& Clarke, V. (2006). Using thematic analysis in psychology. Qualitative Research in Psychology, 3(2), 77-101. doi:10.1191/1478088706qp063oa

Cambpell, E., Sittig, D., Ash, J., Guappone, K., \& Dykstra, R. (2006). Types of unintended consequences related to computerized provider order entry. Journal of the Medical Informatics Association, 13, 547-556.

Canada Health Infoway. (2008). The relationship between electronic health records and patient safety: A joint report on future directions for Canada. Retrieved from https://www.infoway-inforoute.ca/index.php/resources/video-gallery/doc_download/314 $\underline{\text { ehr-patient-safety-report-full }}$

Carrington, J.M. \& Effken, J.A. (2011). Strengths and limitations for the electronic health record for documenting clinical events. CIN: Computers, Informatics, Nursing, 29(6), 360-367 DOI: 10.1097/NCN.0b013e3181fc4139 
Clegg, C. (2000). Sociotechnical principles for system design. Applied Ergonomics, 31(5), 463-477. doi:10.1016/S0003-6870(00)00009-0

Del Baccaro, M., Howard, J., Eisenberg, M., \& Harry, E. (2006). Computerized provider order entry implementation: No association with increased mortality rates in an intensive care unit. Pediatrics , 118, 290-295.

Denzin, N. K., \& Lincoln, Y. S. (2011). The SAGE handbook of qualitative research (4th ed.) Sage.

De Witt, L., \& Ploeg, J. (2006). Critical appraisal of rigour in interpretive phenomenological nursing research. Journal of advanced nursing, 55(2), 215-229.

Dowling, M. (2007). From Husserl to Van Manen. A review of different phenomenological approaches. International Journal of Nursing Studies, 44(1), 131-142. doi:10.1016/j.ijnurstu.2005.11.026

Feng, X., Bobay, K. \& Weiss, M. (2008). Patient safety culture in nursing: A dimensional concept analysis. Journal of Advanced Nursing 63 (3), 310-319 doi: $10.1111 / \mathrm{j} .1365-2648.2008 .04728 . x$

Finlay, L. (2002). Negotiating the swamp: the opportunity and challenge of reflexivity in research practice. Qualitative research, 2(2), 209-230.

Flanagan, M. E., Saleem, J. J., Millitello, L. G., Russ, A. L., \& Doebbeling, B. N. (2013). Paperand computer-based workarounds to electronic health record use at three benchmark institutions. Journal of the American Medical Informatics Association : JAMIA, 20(e1), e59-e66. doi:10.1136/amiajnl-2012-000982 
Han, Y., Carcillo, J., Venkataraman, S., Clark, R., Watson, R., Nguyen, T., et al. (2005).

Unexpected increased mortality after implementation of a commercially sold computerized physician order entry system. Pediatrics , 116, 1506-1512.

Harrington, L., Porch, L., Acosta, K., \& Wilkens, K. (2011). Realizing Electronic Medical Record Benefits. The Journal of Nursing Administration , 41 (7/8), 331-335.

Hassenzahl, M., \& Tractinsky, N. (2006). User experience - a research agenda. Behaviour \& Information Technology, 25(2), 91-97. doi:10.1080/01449290500330331

Hassenzahl, M., \& Ullrich, D. (2007). To do or not to do: Differences in user experience and retrospective judgments depending on the presence or absence of instrumental goals. Interacting with Computers, 19(4), 429-437. doi:10.1016/j.intcom.2007.05.001

Higgins, T., Larson, E., \& Schnall, R. (2017) Unraveling the meaning of patient engagement: A concept analysis, Patient Education and Counseling 100(1), 30-36, doi:10.1016/j.pec.2016.09.002.

Holden, R. J., Carayon, P., Gurses, A. P., Hoonakker, P., Hundt, A. S., Ozok, A. A., \& Rivera Rodriguez, A. J. (2013). SEIPS 2.0: A human factors framework for studying and improving the work of healthcare professionals and patients. Ergonomics, 56(11), 1669 -1686. doi: 10.1080/00140139.2013.838643.

Holden, R. J., \& Karsh, B.-T. (2010). The technology acceptance model: Its past and its future in healthcare. Journal of Biomedical Informatics, 43(1),159-172. doi:10.1016/j.jbi.2009.07.002

Institute for Safe Medication Practices Canada, (n.d.). Medication reconciliation (MedRec) Retreived from: http://www.ismp-canada.org/medrec/\#tab4 
Karsh, B-T., Holden, R.J., Alper, S.J., \& Or, C.K.L. (2006). A human factors engineering paradign for patient safety: Designing to support the performance of the healthcare professional. Quality and Safety in Health Care, 15 (Suppl I), i59-i65.

Keenan, G., Yakel, E., Dunn Lopez, K., Tschannen, D., \& Ford, Y. B. (2013). Challenges to nurses' efforts of retrieving, documenting, and communicating patient care information. Journal of the American Medical Informatics Association : JAMIA, 20(2), 245-251.doi:10.1136/amiajnl-2012-000894

Keohane, C., Bane, A., Featherstone, E., Hayes, J., Woolf, S., Hurley, A., et al. (2008). Quantifying nursing workflow in medication administration. The Journal of Nursing Administration , 38 (1), 19-26.

King, J., Paice, N., Rangrej, J., Forestell, G., \& Swartz, R. (2003). The effect of computerized physician order entry on medication errors and adverse drug events in pediatric inpatients. Pediatrics, 112, 506-509.

Kongsuwan, W., \& Locsin, R. C. (2011). Thai nurses' experience of caring for persons with life sustaining technologies in intensive care settings: A phenomenological study. Intensive \& Critical Care Nursing, 27(2), 102-110. doi:10.1016/j.iccn.2010.12.002

LeGrow, K., Hubley, P., \& McAllister, M. (2010). A conceptual framework for advanced practice nursing in a pediatric tertiary care setting: The SickKids' experience. Nursing Leadership, 23(2), 32-46.

Lincoln, Y. S. (1995). Emerging criteria for quality in qualitative and interpretive research. Qualitative inquiry, 1(3), 275-289. 
Lundvall, L., Dahlgren, M. A., \& Wirell, S. (2014). Professionals' experiences of imaging in the radiography process - A phenomenological approach. Radiography, 20(1), 48-52. doi:10.1016/j.radi.2013.10.002

Manias, E., Gerdtz, M. F., Weiland, T. J., \& Collins, M. (2009). Medication use across transition points from the emergency department: Identifying factors associated with medication discrepancies. The Annals of Pharmacotherapy, 43(11), 1755. doi:10.1345/aph.1M206

Manser, T., \& Foster, S. (2011). Effective handover communication: An overview of research and improvement efforts. Best Practice \& Research Clinical Anaesthesiology,25(2), 181-191. doi:10.1016/j.bpa.2011.02.006

Middleton, B., Bloomrosen, M., Dente, M. A., Hashmat, B., Koppel, R., Overhage, J. M., et al. (2013). Enhancing patient safety and quality of care by improving the usability of electronic health record systems: recommendations from AMIA. Journal of the American Medical Informatics Association, 20(e1), e2-e8.

Morse, J. M. (2000). Determining sample size. Qualitative Health Research, 10(1), 3-5. doi:10.1177/104973200129118183

Northcott, N. (1996). Cognitive mapping: an approach to qualitative data analysis. $N T$ Research, 1(6), 456-463.

Rashotte, J., \& Jensen, L. (2012). The transformational journey of nurse practitioners in acute care. Canadian Journal of Nursing Research,42(2), 70-91.

The Electronic Medication Reconciliation Group. (2014). Paper to electronic MedRec Implementation. Retrieved from Institute for Safe Medication Practices Canada website: http://www.ismp canada.org/download/MedRec/2014_Paper_to_Electronic_MedRec_Implementation_T 
olKit_FINAL.pdf

The Institute of Medicine (1999). To err is human: Building a safer health system. Washington, D.C: National Academy Press.

Thomas, M. J. W., Schultz, T. J., Hannaford, N., \& Runciman, W. B. (2013). Failures in transition: Learning from incidents relating to clinical handover in acute care. Journal for Healthcare Quality, 35(3), 49-56. doi:10.1111/j.1945-1474.2011.00189.x

Tucker, A. L. (2016). The impact of workaround difficulty on frontline employees' response to operational failures: A laboratory experiment on medication administration. Management Science, 62(4), 1124-1144.

Sandelowski, M. (1986). The problem of rigor in qualitative research. Advances in nursing science, 8(3), 27-37.

Sandelowski, M. (1993). Theory unmasked: The uses and guises of theory in qualitative research. Research in nursing \& health, 16(3), 213-218.

Sandelowski, M. (1995). Sample size in qualitative research. Research in Nursing \& Health, 18(2), 179-183. doi:10.1002/nur.4770180211

Schnipper, J. L., Hamann, C., Ndumele, C. D., Liang, C. L., Carty, M. G., Karson, A. S., Gandhi, T. K. (2009). Effect of an electronic medication reconciliation application and process redesign on potential adverse drug events: A cluster-randomized trial. Archives of Internal Medicine, 169(8), 771-780. doi:10.1001/archinternmed.2009.51

Smith, J. A., \& Osborn, M. (2007). Pain as an assault on the self: An interpretative phenomenological analysis of the psychological impact of chronic benign low back pain. Psychology and health, 22(5), 517-534. 
Van Manen, M. (1990). Researching lived experience: Human science for an action sensitive pedagogy. State University of New York Press.

Van Manen, M. (2007). Phenomenology of practice. Phenomenology \& practice, 1(1), 11-30.

Van Manen, M. (2014). Phenomenology of practice: Meaning-giving methods in phenomenological research and writing. Left Coast Press, Inc.

Varpio, L., Schryer, C. F., \& Lingard, L. (2009). Routine and adaptive expert strategies for resolving ICT mediated communication problems in the team setting. Medical education, 43(7), 680-687.

Vishwanath, A., Singh, S. R., \& Winkelstein, P. (2010). The impact of electronic medical record systems on outpatient workflows: A longitudinal evaluation of its workflow effects. International journal of medical informatics, 79(11), 778-791.

Ward, M. M., Vartak, S., Schwichtenberg, T., \& Wakefield, D. S. (2011). Nurses' perceptions of how clinical information system implementation affects workflow and patient care. CIN: Computers, Informatics, Nursing, 29(9), 502-511. doi:10.1097/NCN.0b013e31822b8798

World Health Organization (2008). Summary of the evidence on patient safety: Implications for research. Geneva, Switzerland: WHO Press.

Xiao, Y., Montgomery, D., Philpot, L., Barnes, S., Compton, J., \& Kennerly, D. (2014). Development of a tool to measure user experience following electronic health record implementation. The Journal of Nursing Administration , 44 (7/8), 423-428. 